\title{
ON THE ADDITIVE AND SUBTRACTIVE REPRESENTATION OF EVEN NUMBERS FROM PRIMES
}

\author{
ALI SHEHU ${ }^{1}$ AND JETMIRA UKA ${ }^{2}$ \\ ${ }^{1}$ Queen Mary University of London, United Kingdom \\ a.shehu@qmul.ac.uk \\ 2 Brunel University London, United Kingdom \\ jetmira.uka@brunel.ac.uk
}

\begin{abstract}
We demonstrate a new quantitative method to the sieve of Eratosthenes, which is an alternative to the sieve of Legendre. In this method, every element of a given set is sifted out once only, and therefore, this method is free of the Möbius function and of the consequential parity barrier. Using this method, we prove that every sufficiently large even number is the sum of two primes, and that every even number is the difference of two primes in infinitely many ways.
\end{abstract}

Keywords: Sieve of Eratosthenes, Goldbach's conjecture, Polignac's conjecture, Twin Prime conjecture.

\section{INTRODUCTION}

1.1. Representing even numbers from primes. Since the set of prime numbers is infinite, and since all prime numbers $\geq 3$ are odd numbers, then one knows immediately that there are infinitely many even numbers that can be represented as the sum of two primes, and, infinitely many even numbers that can be represented as the difference of two primes. Having said that, the following questions then naturally arise: (i) Can every even number be represented as the sum of two primes? (ii) Can every even number be represented as the difference of two primes? (iii) Can any even number, or indeed all even numbers, be represented as the difference of two primes, in infinitely many ways? (iv) If questions ' $(i i)^{\prime}$ ' and '( $(i i i)$ ' are answered in the affirmative, can they also hold for consecutive primes, in representing even numbers $\geq 6$ as the difference of two primes?

The earliest known record to have posed question ' $(i)^{\prime}$, known as the Goldbach conjecture, dates back to 1742, in a correspondence between C. Goldbach and L. Euler, where it is propositioned that every even number $>2$ can be represented as the sum of two primes [1] [2]. The Goldbach conjecture has more lately become known as the binary Goldbach conjecture, or the strong Goldbach conjecture, in order to distinguish it from the ternary Goldbach conjecture, or the weak Goldbach conjecture, which states that every odd number $>5$ can be represented as the sum of three primes. The binary Goldbach conjecture has to date been shown empirically to hold for every even number $\leq 4 \cdot 10^{18}[3]$, however, a rigorous proof, or disproof, remains elusive.

Nevertheless, some related theoretical results to the binary Goldbach conjecture have been achieved, the closest of which is due to J. R. Chen, who in 1973, proved that every sufficiently large even number can be represented as the sum of a prime and another prime or a semiprime, that is, the product of at most two primes [4] [5] [6] [7]. On the other hand, significant results have been achieved for the ternary Goldbach conjecture, culminating with a proof given by $\mathrm{H}$. Helfgott in 2014 [8] [9] [10]. 
The earliest known record to have posed questions ' $(i i-i v)^{\prime}$ are due to Alphonse de Polignac, who in 1849, conjectured that every even number can be represented as the difference of two consecutive primes, in infinitely many ways [11]. The most significant special case of Polignac's conjecture, is the so-called 'twin prime conjecture', which comprises of the number 2 being represented as the difference of two primes, in infinitely many ways. The twin prime conjecture is hugely supported by empirical data, where, over the past few decades, increasingly large twin prime pairs have been found to exist [12], with the current world record for a twin prime pair, found in the year 2016, standing at 388, 342 decimal digits long [13].

As with the binary Goldbach Conjecture, the closest theoretical result to Polignac's conjecture is given by J. R. Chen, who in the same article, proved that every even number can be represented as the difference of a prime and another prime or semiprime, that is, the product of at most two primes [7]. More recently, D. A. Goldston, J. Pintz, and C. Y. Yildirim, introduced the now known 'GPY method', which uses approximations to the prime k-tuples conjecture, to study small numbers that can be represented as the difference of two primes [14]. In 2013, Yitang Zhang built on the GPY method, thereby proving for the first time the existence of some even number $<7 \cdot 10^{7}$, which can be represented as the difference of two primes in infinitely many ways [15]. Inspired by Zhang's work, within a year after Zhang first announced his result, J. Maynard presented an independent proof that lowered the bound to 600, which, by assuming the Elliott-Halberstam conjecture, could be further lowered down to 12 [16]. With some refinements to Zhang's method and a combination of that with Maynard's approach, the bound was then lowered to 246 unconditionally, by an on-line collaborative project known as Polymath 8 , organised by T. Tao [17].

1.2. Sieve theory. Sieve theory is a technique for distinguishing specific subsets of integers, amongst the set of natural numbers. Sieve theory began with Eratosthenes of Cyrene (276-194 B.C.), who constructed a method with which one could isolate the subset of prime numbers, from the set of natural numbers [18]. It starts by first crossing the multiples of 2 in the number line up to $x$, then the multiples of 3 , then the multiples of 5 , and then the multiples of all the primes $\leq \sqrt{x}$. If an integer $n>1$ is not divisible by any prime $p \leq \sqrt{x}$, then $n$ is necessarily a prime. Upon completion of the sieve, one has

$$
\#\{P \in \mathbb{P}: P \leq x\}=\#\{n \leq x: P \bigwedge n, P \leq \sqrt{x}\} .
$$

Having the means of constructing the complete subset of prime numbers, from the set of natural numbers up to $x$, one would be naturally interested in quantifying these primes. To this end, there are fundamentally two approaches that one could take, in order to quantify the set of primes generated by the sieve of Eratosthenes. We describe each approach, as follows.

(i) In the first approach, one quantifies the subset of the integers that are sifted out at each round of the sieve, as

$$
\frac{\#\{\text { the subset of all multiples of } P \text { up to } x\}}{\#\{\text { the set of all integers up to } x\}} \text {. }
$$

The easy part with this approach, is that one has no problem in defining quantitatively both the subset of the multiples of $P$ up to $x$ and the set of all integers up to $x$. However, the difficult part is due to the fact that those integers which have multiple prime factors, are necessarily counted at multiple rounds of the sieve, which must be accounted for. This is the approach taken by A. Legendre (1752-1833), who was the first to turn the sieve of Eratosthenes into a quantitative technique, and this has been the approach of choice ever since. In the sieve of Legendre, one counts the integers that are crossed out at each round, and then one subtracts those that are 
counted at multiple rounds, according to the multiplicity of times that this has happened, as follows

$$
[x]-\sum_{P \leq \sqrt{x}}\left[\frac{x}{P}\right]+\sum_{P_{1}<P_{2} \leq \sqrt{x}} \sum_{P_{1} P_{2}}\left[\frac{x}{P_{P_{1}<P_{2}}<P_{3} \leq \sqrt{x}}\left[\frac{x}{P_{1} P_{2} P_{3}}\right]+-\cdots,\right.
$$

from which, one obtains Legendre's formula

$$
\pi(x)-\pi(\sqrt{x})+1=\sum_{\substack{d \\ P \mid d \rightarrow P \leq \sqrt{x}}} \mu(d)\left[\frac{x}{d}\right],
$$

where $\mu(d)$ is the Möbius function, introduced by A. F. Möbius (1790-1868), and defined as

$$
\mu(n):=\left\{\begin{array}{cl}
1 & x=1, \\
1 & x \text { is square-free and has an even number of prime factors, } \\
-1 & x \text { is square-free and has an odd number of prime factors, } \\
0 & x \text { is not square-free }
\end{array}\right.
$$

In efforts to evaluate the right-hand side of (1.3), one has

$$
\pi(x)-\pi(\sqrt{x})+1=x \sum_{d} \frac{\mu(d)}{d}+R=x \prod_{P=P_{1}}^{P \leq \sqrt{x}} \frac{P-1}{P}+R,
$$

where the remainder $R$ is

$$
R=-\sum_{d} \mu(d)\left\{\frac{x}{d}\right\}
$$

which doubles at each round of the sieve, and thus quickly becomes larger than $x$. In modern sieve methods, one tries to replace $\mu(n)$ by a function $\Lambda=\left(\lambda_{d}\right)$, referred to as the "sieve weights", which mimics the $\mu(n)$ and gives satisfactory estimates on upper bounds, lower bounds, and asymptotics for smoother number sets such as almost primes, which leads to upper bounds for primes. However, obtaining lower bounds for primes has proved much more difficult, thus leaving Goldbach's and Polignac's conjectures out of reach. This has been due to the so-called 'parity barrier', where the sieve struggles to distinguish integers with an odd number of prime factors from integers with an even number of prime factors. Although the parity barrier has been broken for certain specific sequences, it still remains the fundamental obstacle in the treatment of primes via this approach of sieve theory.

(ii) In the second approach, one quantifies the subset of the integers that are sifted out at each round of the sieve, as

\#\{the subset of the multiples of $P$ up to $x$ that survived the preceding rounds of the sieve\}

$\#\{$ the set of all the integers up to $x$ that survived the preceding rounds of the sieve\}

This means that every integer is sifted out according to its least prime factor, out of a set of integers whose least prime factors are greater than, or equal to, the least prime factors of the integers being sifted out at the given round, with the exception of the number 1 . This is best illustrated by the following example: In the $1^{\text {st }}$ round of the sieve, one quantifies the subset of the multiples of 2 , as a ratio over the set of all integers, that is

$$
\#\{2,4,6,8,10,12,14,16,18,20,22,24,26\}
$$

$\overline{\#\{1,2,3,4,5,6,7,8,9,10,11,12,13,14,15,16,17,18,19,20,21,22,23,24,25,26\}}$. 
In the $2^{\text {nd }}$ round, one quantifies the subset of the multiples of 3 that survived the preceding round of the sieve, as a ratio over the set of all the integers that survived the preceding round of the sieve, that is

$$
\frac{\#\{3,9,15,21\}}{\#\{1,3,5,7,9,11,13,15,17,19,21,23,25\}} \text {. }
$$

In the $3^{\text {rd }}$ round, one quantifies the subset of the multiples of 5 that survived the preceding rounds of the sieve, as a ratio over the set of all the integers that survived the preceding rounds of the sieve, that is

$$
\frac{\#\{5,25\}}{\#\{1,5,7,11,13,17,19,23,25,\}} .
$$

Since that completes the sieve, then the subset of integers that survive the sieve is

$$
\{1,7,11,13,17,19,23\}
$$

which consists of the number 1 and the complete subset of primes in $[\sqrt{26}, 26]$.

The easy part with this approach, is that every integer is sifted out once only, and so one does not have the problem of certain integers appearing at multiple rounds of the sieve, which one has with the first approach, as described above. However, the difficult part with this approach, is that at each round of the sieve, one finds it difficult to define quantitatively, both the subset of the multiples of $P$ that survive the preceding rounds of the sieve and the set of all the integers that survive the preceding rounds of the sieve. Nevertheless, since at each round of the sieve, the subset of the multiples of $P$ that survive the preceding rounds of the sieve consists of the complete set of integers for which $P$ is the least prime factor, and, the subset of all the integers that survive the preceding rounds of the sieve contains the complete set of primes in $[\sqrt{x}, x]$, then one is hopeful in being able to define quantitatively the two sets of integers, at least to the extent where one can then determine upper and/or lower bounds.

1.3. Our results. In the present paper, we take the second approach as described above, in order to quantify the subset of the integers that survive the sieve of Eratosthenes. On the condition that $x$ is sufficiently large, at each round of the sieve, we define quantitatively both the subset of the multiples of $P$ that survive the preceding rounds of the sieve and the set of all the integers that survive the preceding rounds of the sieve, to the extent where we are then able to determine an upper bound to the subset of the integers that are sifted out at each round of the sieve. To the best of our knowledge, we are the first to take this approach for these purposes, at least to the extent that we do.

Definition 1 (Additive representation). Let $(p, q) \in \mathbb{N}$, let $p \leq q$, and let $x$ be an even number, then we say that $p+q$ is an additive representation of $x$, if $p+q=x$.

Definition 2 (Subtractive representation). Let $(p, q) \in \mathbb{N}$, and let $2 k$ be an even number, then we say that $q-p$ is a subtractive representation of $2 k$, if $q-p=2 k$.

We then extend our approach taken in the sieving of integers, to the sieving of representations, whereby we quantify the subset of the additive representations of $x$, and the subset of the subtractive representations of $2 k$ up to $x$, that survive the sieve of Eratosthenes. As with the sieving of integers, we quantify the subset of the representations that are sifted out at each round of the sieve, as a ratio of the subset of representations containing multiples of $P$ up to $x$ that survived the preceding rounds of the sieve, over the set of representations containing all the integers up to $x$ that survived the preceding rounds of the sieve. This means that additive representations, and subtractive representations, are sifted out according to either $p$ or $q$, depending on whose 
least prime factor is the smaller (or equal to) of the two. This allows for every representation that contains at least one multiple of $P$ to be sifted out once only, while every representation that does not contain multiples of $P$, survives the sieve. Therefore, upon completion of the sieve, we have a subset of representations, where $p=1$ or a prime in $[\sqrt{x}, x]$ and $q$ is a prime in $[\sqrt{x}, x]$, with the quantity of representations where $p=1$ being at most one.

However, contrary to the sieving of integers, in the sieving of additive representations, and in the sieving of subtractive representations, at each round of the sieve, the subset of representations containing multiples of $P$ that survive the preceding rounds of the sieve does not necessarily contain the complete subset of integers for which $P$ is the least prime factor, and, the set of representations containing all the integers that survive the preceding rounds of the sieve does not necessarily contain the complete subset of primes in $[\sqrt{x}, x]$. Therefore, one does not have a set that one can define quantitatively at each round of the sieve, and therefore, one cannot determine an upper bound to the subset of the representations that are sifted out at each round of the sieve, in the same way that can be done for the sieving of integers. Nevertheless, as we demonstrate in this paper, by relating the sieving of representations to the sieving of integers for the same $x$, one can determine an upper bound to the quantity of the additive representations that are sifted out at each round of the sieve, and an upper bound to the quantity of the subtractive representations that are sifted out at each round of the sieve, which we do on the condition that $x$ is sufficiently large. Following on from the upper bounds, we then determine a positive lower bound to the quantity of additive representations that survive the sieve, where both $p$ and $q$ are primes in $[\sqrt{x}, x]$, and a positive lower bound to the quantity of subtractive representations that survive the sieve, where both $p$ and $q$ are primes in $[\sqrt{x}, x]$.

Therefore, we prove the following:

Theorem 1. Every sufficiently large even number is the sum of two prime.

Theorem 2. Every even number is the difference of two primes in infinitely many ways.

Theorem 1 partially addresses question ' $(i)^{\prime}$ as posed above, and, to date is the closest theoretical result to the binary Goldbach conjecture. Theorem 2 fully addresses questions ' $(i i-i i i)^{\prime}$ as posed above, fully establishes the twin prime conjecture, and, to date is the closest theoretical result to Polignac's conjecture.

\section{NotaTion}

$\mathbb{N}$ : the set of natural numbers.

$\mathbb{P}:$ the set of prime numbers.

$P_{1}, P_{2}, P_{3}, P_{4}, P_{70}, P_{71}, P_{m}, P_{n}:$ the $1^{\text {st }}$, the $2^{\text {nd }}$, the $3^{\text {rd }}$, the $4^{\text {th }}$, the $70^{\text {th }}$, the $71^{\text {st }}$, the $m^{\text {th }}$, and the $n^{\text {th }}$ prime numbers respectively. We always have $P_{m}<P_{n}$.

$O_{116}, O_{117}, O_{118}$, and $O_{z}$ : the $116^{\text {th }}, 117^{\text {th }}, 118^{\text {th }}$, and the $z^{\text {th }}$ integers respectively in the sequence $O:\left\{p \in \mathbb{N}: p \leq x, P_{1} \backslash p, P_{2} \backslash p\right\}$.

$x$ and $2 k$ : even numbers.

$p, p_{a}$, and $p_{b}$ : natural numbers, unless explicitly specified.

$w:$ a sufficiently large positive integer, not necessarily the same at every occurrence.

$y_{1}, y_{2}, y_{n}, v_{m}, v_{n}, t_{m}, t_{n}:$ positive real numbers.

$y_{1 a}, y_{2 a}, y_{n a}, y_{1 s}, y_{2 s}, y_{n s}:$ non-negative real numbers.

$a \mid b: a$ divides $b$ evenly, i.e. $b \equiv 0(\bmod a)$.

$a \backslash b: a$ does not divide $b$.

$\beta_{m}:\left\{p \in \mathbb{P}: P_{1} \leq p<P_{m}\right\}$. 
$\beta_{n}:\left\{p \in \mathbb{P}: P_{1} \leq p<P_{n}\right\}$.

$\gamma_{n}:\left\{p \in \mathbb{P}: P_{n}<p \leq \sqrt{x}\right\}$.

$\gamma_{1}:\left\{p \in \mathbb{P}: P_{1}<p \leq \sqrt{x}\right\}$.

$\pi(a): \#\left\{p \in \mathbb{P}: P_{1} \leq p \leq a\right\}$.

$\pi[a, b]: \#\{p \in \mathbb{P}: a \leq p \leq b\}$.

$g(x)^{\prime}: \#\{p \in \mathbb{P}:(x-p) \in \mathbb{P}, p \leq(x-p), \sqrt{x} \leq(p, x-p) \leq x$,$\} .$

$\pi_{2 k}(x)^{\prime}: \#\{p \in \mathbb{P}:(p+2 k) \in \mathbb{P}, \sqrt{x} \leq(p, p+2 k) \leq x\}$.

$\pi_{2 k}(x): \#\left\{p \in \mathbb{P}:(p+2 k) \in \mathbb{P}, P_{1} \leq(p, p+2 k) \leq x\right\}$.

$r_{1} \mathcal{A}: \#\{p \in \mathbb{N}: p \leq x\}$.

$r_{1} \mathcal{A}_{P_{1}}: \#\left\{p \in r_{1} \mathcal{A}: P_{1} \mid p\right\}$.

$r_{1} \mathcal{A}_{P_{n}}: \#\left\{p \in r_{1} \mathcal{A}: P_{n} \mid p\right\}$.

$r_{1} \mathcal{A}_{\gamma_{1}}: \#\left\{p \in r_{1} \mathcal{A}: \gamma_{1} \mid p\right\}$.

$r_{2} \mathcal{A}: \#\left\{p \in r_{1} \mathcal{A}: P_{1} \backslash p\right\}$.

$r_{2} \mathcal{A}_{P_{2}}: \#\left\{p \in r_{2} \mathcal{A}: P_{2} \mid p\right\}$.

$r_{3} \mathcal{A}: \#\left\{p \in r_{2} \mathcal{A}: P_{2} \backslash p\right\}$.

$r_{3} \mathcal{A}_{P_{3}}: \#\left\{p \in r_{3} \mathcal{A}: P_{3} \mid p\right\}$.

$r_{n} \mathcal{A}: \#\left\{p \in r_{1} \mathcal{A}: \beta_{n} \backslash p\right\}$.

$r_{n} \mathcal{A}_{P_{n}}: \#\left\{p \in r_{n} \mathcal{A}: P_{n} \mid p\right\}$.

$r_{n} \mathcal{A}_{\gamma_{n}}: \#\left\{p \in r_{n} \mathcal{A}: \gamma_{n} \mid p\right\}$.

$r_{n+1} \mathcal{A}: \#\left\{p \in r_{n} \mathcal{A}: P_{n} \backslash p\right\}$.

$r_{1} a \mathcal{A}: \#\{p \in \mathbb{N}:(x-p) \in \mathbb{N}, p \leq(x-p)\}$.

$r_{1} a \mathcal{A}_{P_{1}}: \#\left\{p \in r_{1} a \mathcal{A}:(x-p) \in r_{1} a \mathcal{A}, p \leq(x-p), P_{1} \mid p\right.$ and/or $\left.P_{1} \mid(x-p)\right\}$. $r_{1} a \mathcal{A}_{P_{n}}: \#\left\{p \in r_{1} a \mathcal{A}:(x-p) \in r_{1} a \mathcal{A}, p \leq(x-p), P_{n} \mid p\right.$ and/or $\left.P_{n} \mid(x-p)\right\}$. $r_{2} a \mathcal{A}: \#\left\{p \in r_{1} a \mathcal{A}:(x-p) \in r_{1} a \mathcal{A}, p \leq(x-p), P_{1} \backslash p\right.$ and $\left.P_{1} \backslash(x-p)\right\}$.

$r_{2} a \mathcal{A}_{P_{2}}: \#\left\{p \in r_{2} a \mathcal{A}:(x-p) \in r_{2} a \mathcal{A}, p \leq(x-p), P_{2} \mid p\right.$ and/or $\left.P_{2} \mid(x-p)\right\}$. $r_{3} a \mathcal{A}: \#\left\{p \in r_{2} a \mathcal{A}:(x-p) \in r_{2} a \mathcal{A}, p \leq(x-p), P_{2} \backslash p\right.$ and $\left.P_{2} \backslash(x-p)\right\}$.

$r_{3} a \mathcal{A}_{P_{3}}: \#\left\{p \in r_{3} a \mathcal{A}:(x-p) \in r_{3} a \mathcal{A}, p \leq(x-p), P_{3} \mid p\right.$ and/or $\left.P_{3} \mid(x-p)\right\}$.

$r_{n} a \mathcal{A}: \#\left\{p \in r_{1} a \mathcal{A}:(x-p) \in r_{1} a \mathcal{A}, p \leq(x-p), \beta_{n} \backslash p\right.$ and $\left.\beta_{n} \backslash(x-p)\right\}$.

$r_{n} a \mathcal{A}_{P_{n}}: \#\left\{p \in r_{n} a \mathcal{A}:(x-p) \in r_{n} a \mathcal{A}, p \leq(x-p), P_{n} \mid p\right.$ and/or $\left.P_{n} \mid(x-p)\right\}$.

$r_{n+1} a \mathcal{A}: \#\left\{p \in r_{n} a \mathcal{A}:(x-p) \in r_{n} a \mathcal{A}, p \leq(x-p), P_{n} \backslash p\right.$ and $\left.P_{n} \backslash(x-p)\right\}$.

$r_{m} \mathcal{A}: \#\left\{p \in r_{1} \mathcal{A}: \beta_{m} \backslash p\right\}$.

$r_{m} \mathcal{A}_{P_{m}}: \#\left\{p \in r_{m} \mathcal{A}: P_{m} \mid p\right\}$.

$r_{m} \mathcal{A}_{P_{n}}: \#\left\{p \in r_{m} \mathcal{A}: P_{n} \mid p\right\}$.

$r_{m} \mathcal{A}_{P_{m} P_{n}}: \#\left\{p \in r_{m} \mathcal{A}: P_{m} P_{n} \mid p\right\}$.

$r_{m+1} \mathcal{A}: \#\left\{p \in r_{m} \mathcal{A}: P_{m} \backslash p\right\}$.

$r_{m+1} \mathcal{A}_{P_{n}}: \#\left\{p \in r_{m+1} \mathcal{A}: P_{n} \mid p\right\}$.

$r_{m} a \mathcal{A l}: \#\left\{p \in r_{m} \mathcal{A}:(x-p) \in r_{m} \mathcal{A}, p \leq(x-p)\right\}$.

$r_{m} a \mathcal{A}_{P_{m}}: \#\left\{p \in r_{m} a \mathcal{A}:(x-p) \in r_{m} a \mathcal{A}, p \leq(x-p), P_{m} \mid p\right.$ and/or $\left.P_{m} \mid(x-p)\right\}$.

$r_{m} a \mathcal{A}_{P_{n}}: \#\left\{p \in r_{m} a \mathcal{A}:(x-p) \in r_{m} a \mathcal{A}, p \leq(x-p), P_{n} \mid p\right.$ and/or $\left.P_{n} \mid(x-p)\right\}$.

$r_{m} a \mathcal{A}_{P_{m} P_{n}}: \#\left\{p \in r_{m} a \mathcal{A}:(x-p) \in r_{m} a \mathcal{A}, p \leq(x-p), P_{m} \mid p\right.$ and/or $P_{m}\left|(x-p), P_{n}\right| p$ and/or $P_{n} \mid(x-$ p)\}.

$r_{m+1} a \mathcal{A}: \#\left\{p \in r_{m} a \mathcal{A}:(x-p) \in r_{m} a \mathcal{A}, p \leq(x-p), P_{m} \backslash p\right.$ and $\left.P_{m} \backslash(x-p)\right\}$.

$r_{m+1} a \mathcal{A}_{P_{n}}: \#\left\{p \in r_{m+1} a \mathcal{A}:(x-p) \in r_{m+1} a \mathcal{A}, p \leq(x-p), P_{n} \mid p\right.$ and/or $\left.P_{n} \mid(x-p)\right\}$. 
$r_{1} \mathcal{A}^{\prime}: \#\left\{\left(p_{a}, p_{b}\right) \in \mathbb{N}: 1 \leq p_{a} \leq(x-2 k),(2 k+1) \leq p_{b} \leq x\right\}$.

$r_{1} \mathcal{A}_{P_{1}}^{\prime}: \#\left\{\left(p_{a}, p_{b}\right) \in r_{1} \mathcal{A}^{\prime}: P_{1}\left|p_{a}, P_{1}\right| p_{b}\right\}$.

$r_{1} \mathcal{A}_{P_{n}}^{\prime}: \#\left\{\left(p_{a}, p_{b}\right) \in r_{1} \mathcal{A}^{\prime}: P_{n}\left|p_{a}, P_{n}\right| p_{b}\right\}$.

$r_{n} \mathcal{A}^{\prime}: \#\left\{\left(p_{a}, p_{b}\right) \in r_{1} \mathcal{A l}^{\prime}: \beta_{n} \backslash p_{a}, \beta_{n} \backslash p_{b}\right\}$.

$r_{n} \mathcal{A}_{P_{n}}^{\prime}: \#\left\{\left(p_{a}, p_{b}\right) \in r_{n} \mathcal{A}^{\prime}: P_{n}\left|p_{a}, P_{n}\right| p_{b}\right\}$.

$r_{1} s \mathcal{A}^{n}: \#\{p \in \mathbb{N}:(p+2 k) \in \mathbb{N},(p+2 k) \leq x\}$.

$r_{1} s \mathcal{A}_{P_{1}}: \#\left\{p \in r_{1} s \mathcal{A}:(p+2 k) \in r_{1} s \mathcal{A}, P_{1} \mid p\right.$ and/or $\left.P_{1} \mid(p+2 k)\right\}$.

$r_{1} s \mathcal{A}_{P_{n}}: \#\left\{p \in r_{1} s \mathcal{A}:(p+2 k) \in r_{1} s \mathcal{A}, P_{n} \mid p\right.$ and/or $\left.P_{n} \mid(p+2 k)\right\}$.

$r_{2} s \mathcal{A}: \#\left\{p \in r_{1} s \mathcal{A}:(p+2 k) \in r_{1} s \mathcal{A}, P_{1} \backslash p\right.$ and $\left.P_{1} \chi(p+2 k)\right\}$.

$r_{2} s \mathcal{A}_{P_{2}}: \#\left\{p \in r_{2} s \mathcal{A}:(p+2 k) \in r_{2} s \mathcal{A}, P_{2} \mid p\right.$ and/or $\left.P_{2} \mid(p+2 k)\right\}$.

$r_{3} s \mathcal{A}: \#\left\{p \in r_{2} s \mathcal{A}:(p+2 k) \in r_{2} s \mathcal{A}, P_{2} \backslash p\right.$ and $\left.P_{2} \chi(p+2 k)\right\}$.

$r_{3} s \mathcal{A}_{P_{3}}: \#\left\{p \in r_{3} s \mathcal{A}:(p+2 k) \in r_{3} s \mathcal{A}, P_{3} \mid p\right.$ and/or $\left.P_{3} \mid(p+2 k)\right\}$.

$r_{n} s \mathcal{A}: \#\left\{p \in r_{1} s \mathcal{A}:(p+2 k) \in r_{1} s \mathcal{A}, \beta_{n} \backslash p\right.$ and $\left.\beta_{n} \backslash(p+2 k)\right\}$.

$r_{n} s \mathcal{A}_{P_{n}}: \#\left\{p \in r_{n} s \mathcal{A}:(p+2 k) \in r_{n} s \mathcal{A}, P_{n} \mid p\right.$ and/or $\left.P_{n} \mid(p+2 k)\right\}$.

$r_{n+1} s \mathcal{A}: \#\left\{p \in r_{n} s \mathcal{A}:(p+2 k) \in r_{n} s \mathcal{A}, P_{n} \backslash p\right.$ and $\left.P_{n} \backslash(p+2 k)\right\}$.

$r_{m} \mathcal{A}^{\prime}: \#\left\{\left(p_{a}, p_{b}\right) \in r_{1} \mathcal{A}^{\prime}: \beta_{m} \backslash p_{a}, \beta_{m} \bigwedge p_{b}\right\}$.

$r_{m} \mathcal{H}_{P_{m}}^{\prime}: \#\left\{\left(p_{a}, p_{b}\right) \in r_{m} \mathcal{A}^{\prime}: P_{m}\left|p_{a}, P_{m}\right| p_{b}\right\}$.

$r_{m} \mathcal{A}_{P_{n}}^{\prime}: \#\left\{\left(p_{a}, p_{b}\right) \in r_{m} \mathcal{A}^{\prime}: P_{n}\left|p_{a}, P_{n}\right| p_{b}\right\}$.

$r_{m} \mathcal{A}_{P_{m} P_{n}}^{\prime n}: \#\left\{\left(p_{a}, p_{b}\right) \in r_{m} \mathcal{A}^{\prime}: P_{m} P_{n}\left|p_{a}, P_{m} P_{n}\right| p_{b}\right\}$.

$r_{m+1} \mathcal{A}^{\prime}: \#\left\{\left(p_{a}, p_{b}\right) \in r_{m} \mathcal{A}^{\prime}: P_{m} \backslash p_{a}, P_{m} \backslash p_{b}\right\}$.

$r_{m+1} \mathcal{A}_{P_{n}}^{\prime}: \#\left\{\left(p_{a}, p_{b}\right) \in r_{m+1} \mathcal{A}^{\prime}: P_{n}\left|p_{a}, P_{n}\right| p_{b}\right\}$.

$r_{m} s \mathcal{A}: \#\left\{p \in r_{m} \mathcal{A}^{\prime}:(p+2 k) \in r_{m} \mathcal{A}^{\prime}\right\}$.

$r_{m} s \mathcal{A}_{P_{m}}: \#\left\{p \in r_{m} s \mathcal{A}:(p+2 k) \in r_{m} s \mathcal{A}, P_{m} \mid p\right.$ and/or $\left.P_{m} \mid(p+2 k)\right\}$.

$r_{m} s \mathcal{A}_{P_{n}}: \#\left\{p \in r_{m} s \mathcal{A}:(p+2 k) \in r_{m} s \mathcal{A}, P_{n} \mid p\right.$ and/or $\left.P_{n} \mid(p+2 k)\right\}$.

$r_{m} s \mathcal{A}_{P_{m} P_{n}}: \#\left\{p \in r_{m} s \mathcal{A}:(p+2 k) \in r_{m} s \mathcal{A}, P_{m} \mid p\right.$ and/or $P_{m}\left|(p+2 k), P_{n}\right| p$ and/or $\left.P_{n} \mid(p+2 k)\right\}$.

$r_{m+1} s \mathcal{A}: \#\left\{p \in r_{m} s \mathcal{A}:(p+2 k) \in r_{m} s \mathcal{A}, P_{m} \backslash p\right.$ and $\left.P_{m} \backslash(p+2 k)\right\}$.

$r_{m+1} s \mathcal{A}_{P_{n}}: \#\left\{p \in r_{m+1} s \mathcal{A}:(p+2 k) \in r_{m+1} s \mathcal{A}, P_{n} \mid p\right.$ and/or $\left.P_{n} \mid(p+2 k)\right\}$.

\section{Sieve OUtLine}

3.1. Sieving integers. Prior to the $1^{\text {st }}$ round of the sieve, we have

$$
r_{1} \mathcal{A}_{P_{1}}=\frac{y_{1}}{P_{1}} \cdot r_{1} \mathcal{A}
$$

and therefore, at the $1^{\text {st }}$ round of the sieve, we have

$$
S\left(r_{1} \mathcal{A}, P_{1}\right)=r_{1} \mathcal{A}-\frac{y_{1}}{P_{1}} \cdot r_{1} \mathcal{A}=r_{1} \mathcal{A} \cdot \frac{P_{1}-y_{1}}{P_{1}}=r_{2} \mathcal{A} .
$$

Since $r_{1} \mathcal{A}=x$, then

$$
r_{2} \mathcal{A}=x \cdot \frac{P_{1}-y_{1}}{P_{1}}
$$

Prior to the $2^{\text {nd }}$ round of the sieve, we have

$$
r_{2} \mathcal{A}_{P_{2}}=\frac{y_{2}}{P_{2}} \cdot r_{2} \mathcal{A}
$$

and therefore, at the $2^{\text {nd }}$ round of the sieve, we have

$$
S\left(r_{2} \mathcal{A}, P_{2}\right)=r_{2} \mathcal{A}-\frac{y_{2}}{P_{2}} \cdot r_{2} \mathcal{A}=r_{2} \mathcal{A} \cdot \frac{P_{2}-y_{2}}{P_{2}}=r_{3} \mathcal{A},
$$


and therefore

$$
r_{3} \mathcal{A}=x \cdot \frac{P_{1}-y_{1}}{P_{1}} \cdot \frac{P_{2}-y_{2}}{P_{2}} .
$$

Likewise, prior to the $n^{\text {th }}$ round of the sieve, we have

$$
r_{n} \mathcal{A}_{P_{n}}=\frac{y_{n}}{P_{n}} \cdot r_{n} \mathcal{A},
$$

and therefore, at the $n^{\text {th }}$ round of the sieve, we have

$$
S\left(r_{n} \mathcal{A}, P_{n}\right)=r_{n} \mathcal{A}-\frac{y_{n}}{P_{n}} \cdot r_{n} \mathcal{A}=r_{n} \mathcal{A} \cdot \frac{P_{n}-y_{n}}{P_{n}}=r_{n+1} \mathcal{A},
$$

and therefore

$$
r_{n+1} \mathcal{A}=x \cdot \frac{P_{1}-y_{1}}{P_{1}} \cdot \frac{P_{2}-y_{2}}{P_{2}} \cdots \cdot \frac{P_{n}-y_{n}}{P_{n}} .
$$

Since, upon completion of the sieve, we have

$$
S\left(r_{n} \mathcal{A}, P_{n}, \sqrt{x}\right)=\pi(x)-\pi(\sqrt{x})+1,
$$

then

$$
\pi(x)-\pi(\sqrt{x})+1=x \cdot \prod_{P_{n}=P_{1}}^{P_{n} \leq \sqrt{x}} \frac{P_{n}-y_{n}}{P_{n}} .
$$

Empirical data, as shown in Figure 1, suggest that $y_{n}$ oscillates about $y_{n}=1$, before tending to zero as $P_{n} \rightarrow \sqrt{x}$. In section 4, we determine an upper bound to $y_{n}$, and therefore to (3.7), for $x \geq w$ and $P_{n} \leq \sqrt{x}$.

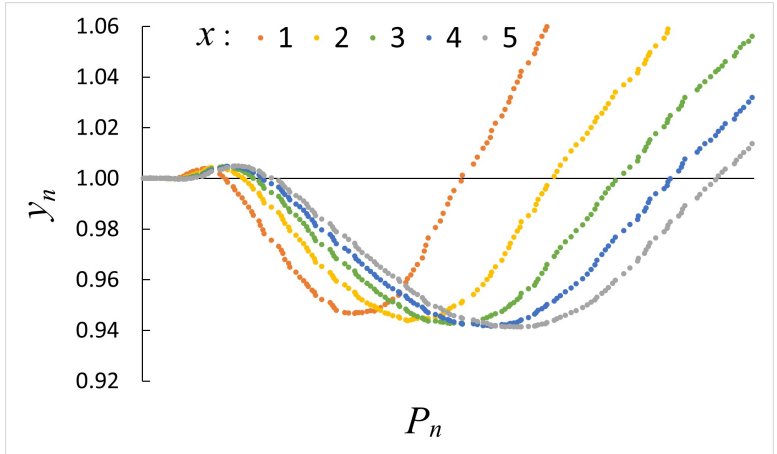

(a) $P_{1} \leq P_{n} \leq 1 \cdot 10^{3}$

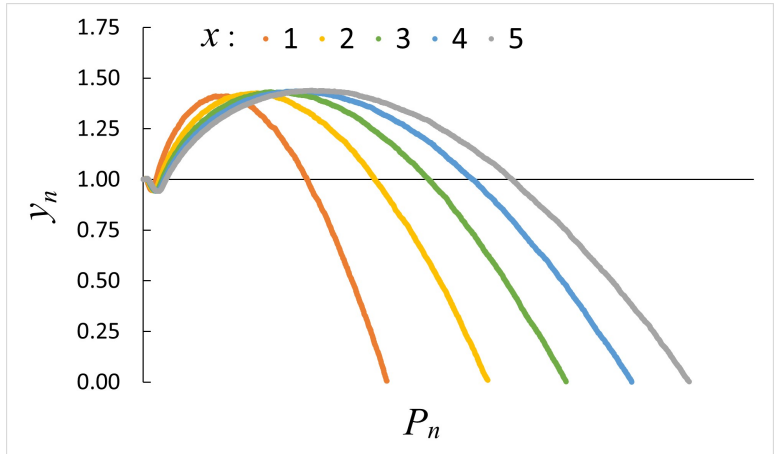

(b) $P_{1} \leq P_{n} \leq \sqrt{x}$

FIGURE 1. $\frac{r_{n} \mathcal{A}_{P_{n}}}{r_{n} \mathcal{A}}=\frac{1}{P_{n}} \cdot y_{n}$, for various $x \cdot 10^{8}$

3.2. Sieving additive representations. Prior to the $1^{\text {st }}$ round of the sieve, we have

$$
r_{1} a \mathcal{A}_{P_{1}}=\frac{y_{1 a}}{P_{1}} \cdot r_{1} a \mathcal{A},
$$

and therefore, at the $1^{\text {st }}$ round of the sieve, we have

$$
S\left(r_{1} a \mathcal{A}, P_{1}\right)=r_{1} a \mathcal{A}-\frac{y_{1 a}}{P_{1}} \cdot r_{1} a \mathcal{A}=r_{1} a \mathcal{A} \cdot \frac{P_{1}-y_{1 a}}{P_{1}}=r_{2} a \mathcal{A} .
$$


Since $r_{1} a \mathcal{A}=\frac{x}{2}$, then

$$
r_{2} a \mathcal{A}=\frac{x}{2} \cdot \frac{P_{1}-y_{1 a}}{P_{1}}
$$

Prior to the $2^{\text {nd }}$ round of the sieve, we have

$$
r_{2} a \mathcal{A}_{P_{2}}=\frac{y_{2 a}}{P_{2}} \cdot r_{2} a \mathcal{A}
$$

and therefore, at the $2^{\text {nd }}$ round of the sieve, we have

$$
S\left(r_{2} a \mathcal{A}, P_{2}\right)=r_{2} a \mathcal{A}-\frac{y_{2 a}}{P_{2}} \cdot r_{2} a \mathcal{A}=r_{2} a \mathcal{A} \cdot \frac{P_{2}-y_{2 a}}{P_{2}}=r_{3} a \mathcal{A},
$$

and therefore

$$
r_{3} a \mathcal{A}=\frac{x}{2} \cdot \frac{P_{1}-y_{1 a}}{P_{1}} \cdot \frac{P_{2}-y_{2 a}}{P_{2}}
$$

Likewise, prior to the $n^{\text {th }}$ round of the sieve, we have

$$
r_{n} a \mathcal{A}_{P_{n}}=\frac{y_{n a}}{P_{n}} \cdot r_{n} a \mathcal{A},
$$

and therefore, at the $n^{\text {th }}$ round of the sieve, we have

$$
S\left(r_{n} a \mathcal{A}, P_{n}\right)=r_{n} a \mathcal{A}-\frac{y_{n a}}{P_{n}} \cdot r_{n} a \mathcal{A}=r_{n} a \mathcal{A} \cdot \frac{P_{n}-y_{n a}}{P_{n}}=r_{n+1} a \mathcal{A},
$$

and therefore

$$
r_{n+1} a \mathcal{A}=\frac{x}{2} \cdot \frac{P_{1}-y_{1 a}}{P_{1}} \cdot \frac{P_{2}-y_{2 a}}{P_{2}} \cdots \frac{P_{n}-y_{n a}}{P_{n}} .
$$

Since, upon completion of the sieve, we have

$$
S\left(r_{n} a \mathcal{A}, P_{n}, \sqrt{x}\right)=g(x)^{\prime}+u_{a}
$$

where $u_{a}=1$ or 0 , then

$$
g(x)^{\prime} \geq \frac{x}{2} \cdot \prod_{P_{n}=P_{1}}^{P_{n} \leq \sqrt{x}} \frac{P_{n}-y_{n a}}{P_{n}}-1
$$

Empirical data for the sieving of additive representations, as shown in Figure 2 for all $P_{n} \leq$ $\sqrt{x}$, suggest that

$$
\frac{r_{n} a \mathcal{A}_{P_{n}}}{r_{n} a \mathcal{A}} \approx \begin{cases}1 \cdot \frac{r_{n} \mathcal{A}_{P_{n}}}{r_{n} \mathcal{A}}, & \text { if } P_{n} \mid x, \\ 2 \cdot \frac{r_{n} \mathcal{A}_{P_{n}}}{r_{n} \mathcal{A}}, & \text { if } P_{n} \backslash x,\end{cases}
$$

which, in section 5 we generalise for all $x$ and $P_{n} \leq \sqrt{x}$. Through (3.23), we are able to determine upper bounds to $y_{n a}$, and therefore to (3.18), which we do in section 5 for $x \geq w$ and $P_{n} \leq \sqrt{x}$; which then allows us to determine a positive lower bound to (3.22) for $x \geq w$, which we do in section 7 , and thereby we prove theorem 1 . 


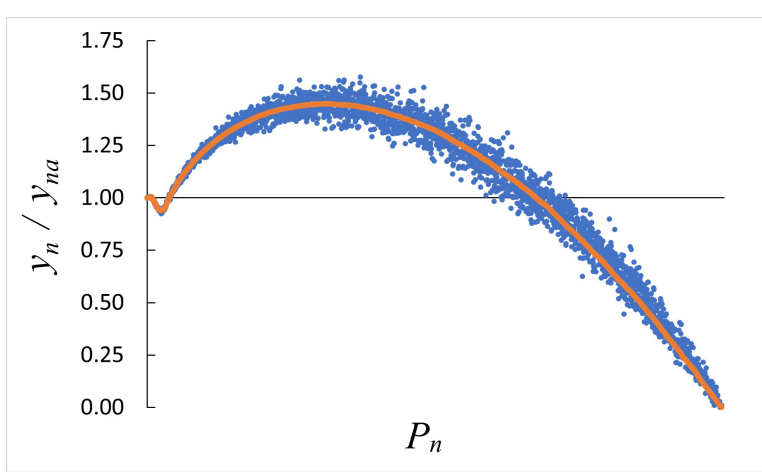

(a) $x=892,371,464$

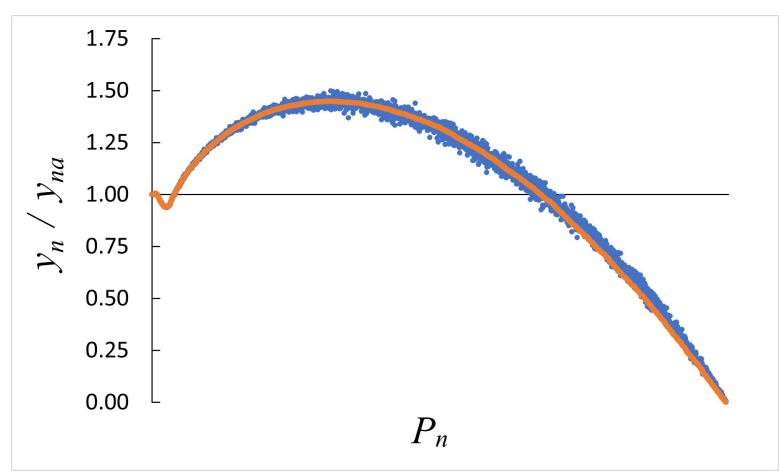

(b) $x=892,371,480$

$$
\text { FIGURE 2. } \frac{r_{n} \mathcal{A}_{P_{n}}}{r_{n} \mathcal{A}}=\frac{1}{P_{n}} \cdot y_{n}(\mathrm{red}) \text { and } \frac{r_{n} a \mathcal{A}_{P_{n}}}{r_{n} a \mathcal{A}}=\left\{\begin{array}{ll}
\frac{1}{P_{n}} \cdot y_{n a}, & \text { if } P_{n} \mid x, \\
\frac{2}{P_{n}} \cdot y_{n a}, & \text { if } P_{n} \backslash x,
\end{array}\right. \text { (blue). }
$$

3.3. Sieving subtractive representations. Prior to the $1^{\text {st }}$ round of the sieve, we have

$$
r_{1} s \mathcal{A}_{P_{1}}=\frac{y_{1 s}}{P_{1}} \cdot r_{1} s \mathcal{A},
$$

and therefore, at the $1^{\text {st }}$ round of the sieve, we have

$$
S\left(r_{1} s \mathcal{A}, P_{1}\right)=r_{1} s \mathcal{A}-\frac{y_{1 s}}{P_{1}} \cdot r_{1} s \mathcal{A}=r_{1} s \mathcal{A} \cdot \frac{P_{1}-y_{1 s}}{P_{1}}=r_{2} s \mathcal{A} .
$$

Since $r_{1} s \mathcal{A}=x-2 k$, then

$$
r_{2} s \mathcal{A}=(x-2 k) \cdot \frac{P_{1}-y_{1 s}}{P_{1}} .
$$

Prior to the $2^{\text {nd }}$ round of the sieve, we have

$$
r_{2} s \mathcal{A}_{P_{2}}=\frac{y_{2 s}}{P_{2}} \cdot r_{2} s \mathcal{A},
$$

and therefore, at the $2^{\text {nd }}$ round of the sieve, we have

$$
S\left(r_{2} s \mathcal{A}, P_{2}\right)=r_{2} s \mathcal{A}-\frac{y_{2 s}}{P_{2}} \cdot r_{2} s \mathcal{A}=r_{2} s \mathcal{A} \cdot \frac{P_{2}-y_{2 s}}{P_{2}}=r_{3} s \mathcal{A},
$$

and therefore

$$
r_{3} s \mathcal{A}=(x-2 k) \cdot \frac{P_{1}-y_{1 s}}{P_{1}} \cdot \frac{P_{2}-y_{2 s}}{P_{2}} .
$$

Likewise, prior to the $n^{\text {th }}$ round of the sieve, we have

$$
r_{n} s \mathcal{A}_{P_{n}}=\frac{y_{n s}}{P_{n}} \cdot r_{n} s \mathcal{A},
$$

and therefore, at the $n^{\text {th }}$ round of the sieve, we have

$$
S\left(r_{n} s \mathcal{A}, P_{n}\right)=r_{n} s \mathcal{A}-\frac{y_{n s}}{P_{n}} \cdot r_{n} s \mathcal{A}=r_{n} s \mathcal{A} \cdot \frac{P_{n}-y_{n s}}{P_{n}}=r_{n+1} s \mathcal{A},
$$

and therefore

$$
r_{n+1} s \mathcal{A}=(x-2 k) \cdot \frac{P_{1}-y_{1 s}}{P_{1}} \cdot \frac{P_{2}-y_{2 s}}{P_{2}} \cdots \frac{P_{n}-y_{n s}}{P_{n}} .
$$


Since, upon completion of the sieve, we have

$$
S\left(r_{n} S \mathcal{A}, P_{n}, \sqrt{x}\right)=\pi_{2 k}(x)^{\prime}+u_{s},
$$

where $u_{s}=1$ or 0 , then

$$
\pi_{2 k}(x)^{\prime} \geq(x-2 k) \cdot \prod_{P_{n}=P_{1}}^{P_{n} \leq \sqrt{x}} \frac{P_{n}-y_{n s}}{P_{n}}-1 .
$$

As with the sieving of additive representations, empirical data for the sieving of subtractive representations, as shown in Figure 3 for all $P_{n} \leq \sqrt{x}$, suggest that

$$
\frac{r_{n} s \mathcal{A}_{P_{n}}}{r_{n} s \mathcal{A}} \approx \begin{cases}1 \cdot \frac{r_{n} \mathcal{A}_{P_{n}}}{r_{n} \mathcal{A}}, & \text { if } P_{n} \mid 2 k \\ 2 \cdot \frac{r_{n} \mathcal{A}_{P_{n}}}{r_{n} \mathcal{A}}, & \text { if } P_{n} \backslash 2 k\end{cases}
$$

which, in section 6, we generalise for all $x$ and $P_{n} \leq \sqrt{x}$. Through (3.35), we are able to determine upper bounds to $y_{n s}$, and therefore to (3.30), which we do in section 6 for $x \geq w$ and $P_{n} \leq \sqrt{x}$; which then allows us to determine a positive lower bound to (3.34) for $x \geq w$, which we do in section 7, and which then implies that $\pi_{2 k}(x) \rightarrow \infty$ as $x \rightarrow \infty$, and thereby we prove theorem 2.

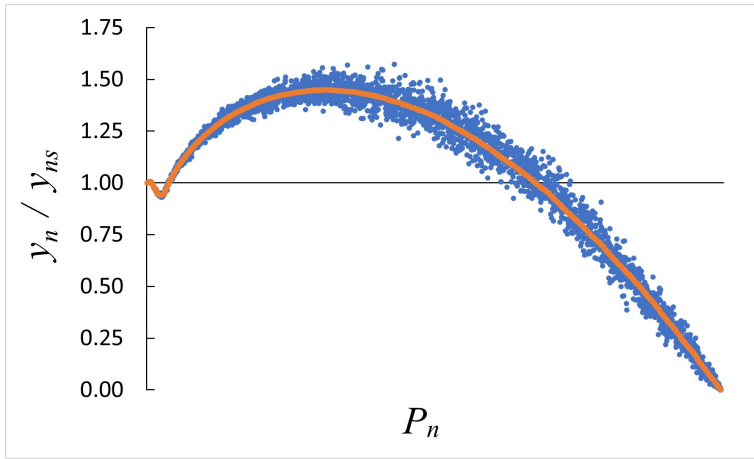

(a) $x=892,371,464$ and $2 k=446,185,732$

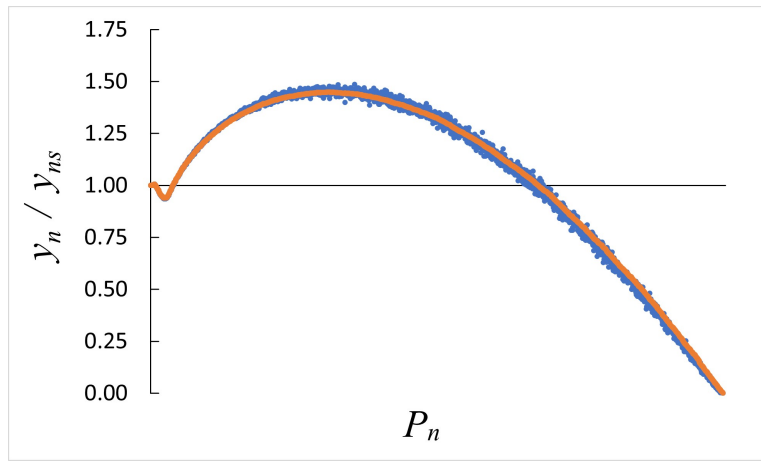

(b) $x=892,371,464$ and $2 k=9,699,690$ FIGURE 3. $\frac{r_{n} \mathcal{A}_{P_{n}}}{r_{n} \mathcal{A}}=\frac{1}{P_{n}} \cdot y_{n}$ (red) and $\frac{r_{n} s \mathcal{A}_{P_{n}}}{r_{n} s \mathcal{A}}=\left\{\begin{array}{ll}\frac{1}{P_{n}} \cdot y_{n s}, & \text { if } P_{n} \mid 2 k, \\ \frac{2^{2}}{P_{n}} \cdot y_{n s}, & \text { if } P_{n} \backslash 2 k,\end{array}\right.$ (blue).

\section{AN UPPER BOUND ON THE SIFTED OUT INTEGERS}

In this section, we determine an upper bound to (3.7), as follows. Prior to the $n^{\text {th }}$ round of the sieve, we have

$$
\frac{r_{n} \mathcal{A}_{P_{n}}}{r_{n} \mathcal{A}}=\frac{r_{n} \mathcal{A}_{P_{n}}}{r_{n} \mathcal{A}_{P_{n}}+r_{n} \mathcal{A}_{\gamma_{n}}+\pi\left[P_{n}, x\right]+1} \leq \frac{r_{n} \mathcal{A}_{P_{n}}}{r_{n} \mathcal{A}_{P_{n}}+r_{n} \mathcal{A}_{\gamma_{n}}+\pi\left[P_{n}, x\right]} .
$$

Since

$$
\pi\left(P_{n-1}\right)+\pi\left[P_{n}, x\right]=\pi(x),
$$


and

$$
\frac{\pi\left(P_{n-1}\right)}{r_{n} \mathcal{A}_{P_{n}}+r_{n} \mathcal{A}_{\gamma_{n}}+\pi\left[P_{n}, x\right]} \leq \frac{\pi\left(P_{n-1}\right)}{r_{n} \mathcal{A}_{P_{n}}}
$$

then

$$
\frac{r_{n} \mathcal{A}_{P_{n}}}{r_{n} \mathcal{A}_{P_{n}}+r_{n} \mathcal{A}_{\gamma_{n}}+\pi\left[P_{n}, x\right]} \leq \frac{r_{n} \mathcal{A}_{P_{n}}+\pi\left(P_{n-1}\right)}{r_{n} \mathcal{A}_{P_{n}}+r_{n} \mathcal{A}_{\gamma_{n}}+\pi(x)} .
$$

Since $r_{n} \mathcal{A}_{P_{n}}$ consists of the complete subset of integers for which $P_{n}$ is the least prime factor, then due to the Fundamental Theorem of Arithmetic, we have

$$
r_{n} \mathcal{A}_{P_{n}}=\pi\left[P_{n}, \frac{x}{P_{n}}\right]+D
$$

where $D \rightarrow 0$ as $P_{n} \rightarrow \sqrt[3]{x}$, and $r_{n} \mathcal{A}_{P_{n}} \rightarrow \pi\left[P_{n}, \frac{x}{P_{n}}\right]$ as $P_{n} \rightarrow \sqrt[3]{x}$, and therefore

$$
D= \begin{cases}r_{n} \mathcal{A}_{P_{n}}-\pi\left[P_{n}, \frac{x}{P_{n}}\right] \geq 1, & \text { if } P_{1} \leq P_{n} \leq \sqrt[3]{x} \\ 0, & \text { if } \sqrt[3]{x}<P_{n} \leq \sqrt{x}\end{cases}
$$

Since

$$
\pi\left(P_{n-1}\right)+\pi\left[P_{n}, \frac{x}{P_{n}}\right]=\pi\left(\frac{x}{P_{n}}\right)
$$

then, due to (4.5), we have

$$
r_{n} \mathcal{A}_{P_{n}}+\pi\left(P_{n-1}\right)=\pi\left(\frac{x}{P_{n}}\right)+D
$$

and, due to (4.6), we have

$$
D= \begin{cases}r_{n} \mathcal{A}_{P_{n}}+\pi\left(P_{n-1}\right)-\pi\left(\frac{x}{P_{n}}\right) \geq 1, & \text { if } P_{1} \leq P_{n} \leq \sqrt[3]{x} \\ 0, & \text { if } \sqrt[3]{x}<P_{n} \leq \sqrt{x}\end{cases}
$$

and therefore

$$
\frac{D}{\pi\left(\frac{x}{P_{n}}\right)+D}=\frac{r_{n} \mathcal{A}_{P_{n}}+\pi\left(P_{n-1}\right)-\pi\left(\frac{x}{P_{n}}\right)}{r_{n} \mathcal{A}_{P_{n}}+\pi\left(P_{n-1}\right)}
$$

If $P_{n}=P_{1}$, then

$$
\frac{r_{1} \mathcal{A}_{P_{1}}+\pi\left(P_{1-1}\right)-\pi\left(\frac{x}{P_{1}}\right)}{r_{1} \mathcal{A}_{P_{1}}+\pi\left(P_{1-1}\right)}=\frac{\frac{x}{2}-\pi\left(\frac{x}{2}\right)}{\frac{x}{2}}
$$

and, if $\sqrt[3]{x}<P_{n} \leq \sqrt{x}$, then

$$
\frac{r_{n} \mathcal{A}_{P_{n}}+\pi\left(P_{n-1}\right)-\pi\left(\frac{x}{P_{n}}\right)}{r_{n} \mathcal{A}_{P_{n}}+\pi\left(P_{n-1}\right)}=0 .
$$


If $P_{1} \leq P_{n} \leq \sqrt[3]{x}$, then

$$
\lim _{P_{n} \rightarrow \sqrt[3]{x}}\left(r_{n} \mathcal{A}_{P_{n}}+\pi\left(P_{n-1}\right)-\pi\left(\frac{x}{P_{n}}\right)\right)=0
$$

and

$$
\lim _{P_{n} \rightarrow \sqrt[3]{x}}\left(r_{n} \mathcal{A}_{P_{n}}+\pi\left(P_{n-1}\right)\right)=\pi\left(\frac{x}{\sqrt[3]{x}}\right)
$$

and therefore

$$
\lim _{P_{n} \rightarrow \sqrt[3]{x}} \frac{r_{n} \mathcal{A}_{P_{n}}+\pi\left(P_{n-1}\right)-\pi\left(\frac{x}{P_{n}}\right)}{r_{n} \mathcal{A}_{P_{n}}+\pi\left(P_{n-1}\right)}=0 .
$$

Furthermore, if $P_{n}=P_{1}$, then

$$
\frac{r_{1} \mathcal{A}_{P_{1}}+r_{1} \mathcal{A}_{\gamma_{1}}}{r_{1} \mathcal{A}_{P_{1}}+r_{1} \mathcal{A}_{\gamma_{1}}+\pi(x)}=\frac{x-\pi(x)}{x} .
$$

If $P_{1} \leq P_{n} \leq \sqrt{x}$, then

$$
\lim _{P_{n} \rightarrow \sqrt{x}} \frac{r_{n} \mathcal{A}_{P_{n}}+r_{n} \mathcal{A}_{\gamma_{n}}}{r_{n} \mathcal{A}_{P_{n}}+r_{n} \mathcal{A}_{\gamma_{n}}+\pi(x)}=0
$$

and therefore, if $P_{1} \leq P_{n} \leq \sqrt[3]{x}$, then

$$
\lim _{P_{n} \rightarrow \sqrt[3]{x}} \frac{r_{n} \mathcal{A}_{P_{n}}+r_{n} \mathcal{A}_{\gamma_{n}}}{r_{n} \mathcal{A}_{P_{n}}+r_{n} \mathcal{A}_{\gamma_{n}}+\pi(x)}=h>0 .
$$

Since $\frac{\sqrt{x}}{\sqrt[3]{x}} \rightarrow \infty$ as $x \rightarrow \infty$, then $h \rightarrow\left(\frac{x-\pi(x)}{x}\right)$ as $x \rightarrow \infty$.

Therefore, if $x \geq w$ and $P_{1} \leq P_{n} \leq \sqrt{x}$, then

$$
\frac{r_{n} \mathcal{A}_{P_{n}}+\pi\left(P_{n-1}\right)-\pi\left(\frac{x}{P_{n}}\right)}{r_{n} \mathcal{A}_{P_{n}}+\pi\left(P_{n-1}\right)} \leq \frac{r_{n} \mathcal{A}_{P_{n}}+r_{n} \mathcal{A}_{\gamma_{n}}}{r_{n} \mathcal{A}_{P_{n}}+r_{n} \mathcal{A}_{\gamma_{n}}+\pi(x)},
$$

and therefore, from the right-hand side of (4.4), we have

$$
\frac{r_{n} \mathcal{A}_{P_{n}}+\pi\left(P_{n-1}\right)}{r_{n} \mathcal{A}_{P_{n}}+r_{n} \mathcal{A}_{\gamma_{n}}+\pi(x)} \leq \frac{r_{n} \mathcal{A}_{P_{n}}+\pi\left(P_{n-1}\right)-\left(r_{n} \mathcal{A}_{P_{n}}+\pi\left(P_{n-1}\right)-\pi\left(\frac{x}{P_{n}}\right)\right)}{r_{n} \mathcal{A}_{P_{n}}+r_{n} \mathcal{A}_{\gamma_{n}}+\pi(x)-\left(r_{n} \mathcal{A}_{P_{n}}+r_{n} \mathcal{A}_{\gamma_{n}}\right)},
$$

and therefore

$$
\frac{r_{n} \mathcal{A}_{P_{n}}}{r_{n} \mathcal{A}} \leq \frac{\pi\left(\frac{x}{P_{n}}\right)}{\pi(x)}
$$

which we quantify as follows.

Let

$$
f\left(\frac{x}{P_{n}}\right)=\frac{\pi\left(\frac{x}{P_{n}}\right)}{\frac{x}{P_{n}}}
$$


and

$$
f(x)=\frac{\pi(x)}{x}
$$

then

$$
\frac{\pi\left(\frac{x}{P_{n}}\right)}{\pi(x)}=\frac{1}{P_{n}} \cdot \frac{f\left(\frac{x}{P_{n}}\right)}{f(x)} .
$$

Let

$$
f(\sqrt{x})=\frac{\pi(\sqrt{x})}{\sqrt{x}}
$$

then

$$
\frac{f\left(\frac{x}{P_{n}}\right)}{f(x)} \leq \frac{f(\sqrt{x})}{f(x)}
$$

Due to the Prime Number Theorem, we have

$$
\lim _{x \rightarrow \infty} \frac{f(\sqrt{x})}{f(x)}=2,
$$

with the limit being approached from above. Therefore, if $d>2$ and $x \geq w$, then

$$
\frac{f(\sqrt{x})}{f(x)} \leq d \text {. }
$$

Therefore, if $x \geq w$ and $P_{n} \leq \sqrt{x}$, then

$$
\frac{r_{n} \mathcal{A}_{P_{n}}}{r_{n} \mathcal{A}} \leq \frac{1}{P_{n}} \cdot 2.15
$$

and thus, we have determined an upper bound to (3.7).

\section{UPPER BOUNDS ON THE SIFTED OUT ADDITIVE REPRESENTATIONS}

In this section, we determine upper bounds to (3.18), as follows. Prior to the $1^{\text {st }}$ round of the sieve, we have

$$
r_{1} a \mathcal{A}=\frac{1}{2} \cdot r_{1} \mathcal{A}
$$

and

$$
\begin{cases}\text { if } P_{n} \mid x \text { and } P_{n} \mid p, & \text { then } P_{n} \mid(x-p), \\ \text { if } P_{n} \backslash x \text { and } P_{n} \mid p, & \text { then } P_{n} \backslash(x-p), \\ \text { if } P_{n} \backslash x \text { and } P_{n} \mid(x-p), & \text { then } P_{n} \backslash p,\end{cases}
$$

and therefore

$$
r_{1} a \mathcal{A}_{P_{n}}= \begin{cases}\frac{1}{2} \cdot r_{1} \mathcal{A}_{P_{n}}, & \text { if } P_{n} \mid x \\ \frac{1}{1} \cdot r_{1} \mathcal{A}_{P_{n}}, & \text { if } P_{n} \backslash x\end{cases}
$$


Due to (5.1) and (5.3), we have

$$
\frac{r_{1} a \mathcal{A}_{P_{n}}}{r_{1} a \mathcal{A}}= \begin{cases}1 \cdot \frac{r_{1} \mathcal{A}_{P_{n}}}{r_{1} \mathcal{A}}, & \text { if } P_{n} \mid x, \\ 2 \cdot \frac{r_{1} \mathcal{A}_{P_{n}}}{r_{1} \mathcal{A}}, & \text { if } P_{n} \backslash x .\end{cases}
$$

In the following, we describe the sieving process of additive representations at the $m^{\text {th }}$ round of the sieve, in comparison to the sieving process of integers, also at the $m^{\text {th }}$ round of the sieve. The $m^{\text {th }}$ round of the sieve precedes the $n^{\text {th }}$ round of the sieve. Thereby, we show that (5.4) is almost preserved prior to the $n^{\text {th }}$ round of the sieve, hence generalising (3.23).

Prior to the $m^{\text {th }}$ round of the sieve, we have

$$
r_{m} \mathcal{A}_{P_{m}}=\frac{v_{m}}{P_{m}} \cdot r_{m} \mathcal{A}
$$

and

$$
r_{m} \mathcal{A}_{P_{n}}=\frac{v_{n}}{P_{n}} \cdot r_{m} \mathcal{A},
$$

and therefore

$$
r_{m} \mathcal{A}_{P_{m} P_{n}}=\frac{v_{m}}{P_{m}} \cdot \frac{v_{n}}{P_{n}} \cdot r_{m} \mathcal{A},
$$

and therefore

$$
\frac{v_{n}}{P_{n}} \cdot r_{m} \mathcal{A}_{P_{m}} \approx \frac{v_{m}}{P_{m}} \cdot r_{m} \mathcal{A}_{P_{n}} .
$$

When sieving additive representations, prior to the $n^{\text {th }}$ round of the sieve, we have

$$
\begin{cases}\text { if } p \in r_{n} a \mathcal{A}, & \text { then }(x-p) \in r_{n} a \mathcal{A}, \\ \text { if }(x-p) \in r_{n} a \mathcal{A}, & \text { then } p \in r_{n} a \mathcal{A},\end{cases}
$$

and therefore, we assume that

$$
\begin{cases}\text { if } p \in r_{m} \mathcal{A}, & \text { then }(x-p) \in r_{m} \mathcal{A}, \\ \text { if }(x-p) \in r_{m} \mathcal{A}, & \text { then } p \in r_{m} \mathcal{A} .\end{cases}
$$

Thus, we have

$$
r_{m} a \mathcal{A}=\frac{1}{2} \cdot r_{m} \mathcal{A},
$$

and

$$
\begin{cases}\text { if } P_{m} \mid x \text { and } P_{m} \mid p, & \text { then } P_{m} \mid(x-p), \\ \text { if } P_{m} \backslash x \text { and } P_{m} \mid p, & \text { then } P_{m} \backslash(x-p), \\ \text { if } P_{m} \backslash x \text { and } P_{m} \mid(x-p), & \text { then } P_{m} \backslash p,\end{cases}
$$

and therefore

$$
r_{m} a \mathcal{A}_{P_{m}}= \begin{cases}\frac{1}{2} \cdot r_{m} \mathcal{A}_{P_{m}}, & \text { if } P_{m} \mid x, \\ \frac{1}{1} \cdot r_{m} \mathcal{A}_{P_{m}}, & \text { if } P_{m} \backslash x,\end{cases}
$$


and

$$
\begin{cases}\text { if } P_{n} \mid x \text { and } P_{n} \mid p, & \text { then } P_{n} \mid(x-p), \\ \text { if } P_{n} \backslash x \text { and } P_{n} \mid p, & \text { then } P_{n} \backslash(x-p), \\ \text { if } P_{n} \backslash x \text { and } P_{n} \mid(x-p), & \text { then } P_{n} \backslash p,\end{cases}
$$

and therefore

$$
r_{m} a \mathcal{A}_{P_{n}}= \begin{cases}\frac{1}{2} \cdot r_{m} \mathcal{A}_{P_{n}}, & \text { if } P_{n} \mid x, \\ \frac{1}{1} \cdot r_{m} \mathcal{A}_{P_{n}}, & \text { if } P_{n} \backslash x\end{cases}
$$

Due to (5.5), (5.11), and (5.13), we have

$$
r_{m} a \mathcal{A}_{P_{m}}= \begin{cases}\frac{v_{m}}{P_{m}} \cdot r_{m} a \mathcal{A}, & \text { if } P_{m} \mid x, \\ \frac{2 v_{m}}{P_{m}} \cdot r_{m} a \mathcal{A}, & \text { if } P_{m} \backslash x .\end{cases}
$$

Therefore, if we sieve integers, then due to (5.5), at the $m^{\text {th }}$ round of the sieve, we have

$$
S\left(r_{m} \mathcal{A}, P_{m}\right)=r_{m} \mathcal{A} \cdot\left(\frac{P_{m}-v_{m}}{P_{m}}\right),
$$

and, if we sieve additive representations, then due to $(5.16)$, at the $m^{\text {th }}$ round of the sieve, we have

$$
S\left(r_{m} a \mathcal{A}, P_{m}\right)= \begin{cases}r_{m} a \mathcal{A} \cdot\left(\frac{P_{m}-v_{m}}{P_{m}}\right), & \text { if } P_{m} \mid x, \\ r_{m} a \mathcal{A} \cdot\left(\frac{P_{m}-2 v_{m}}{P_{m}}\right), & \text { if } P_{m} \backslash x .\end{cases}
$$

Furthermore, due to (5.5), (5.6), (5.11), (5.13), and (5.15), we have

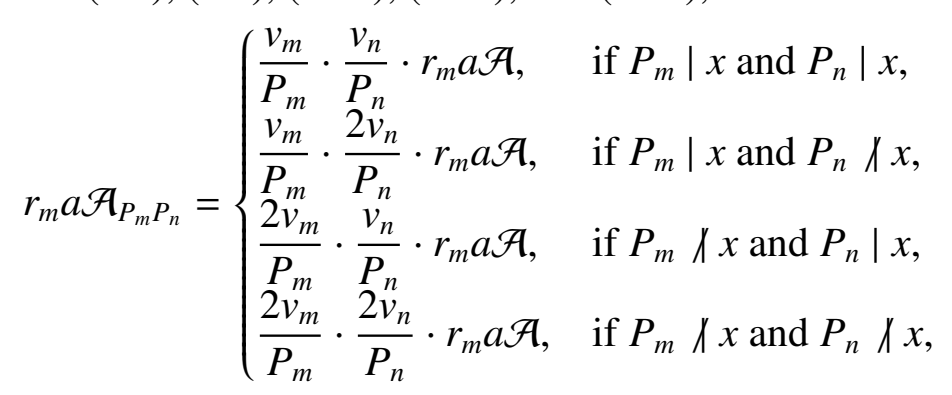

and therefore

$$
r_{m} a \mathcal{A}_{P_{m} P_{n}} \approx \begin{cases}\frac{v_{n}}{P_{n}} \cdot r_{m} a \mathcal{A}_{P_{m}} \approx \frac{v_{m}}{P_{m}} \cdot r_{m} a \mathcal{A}_{P_{n}}, & \text { if } P_{m} \mid x \text { and } P_{n} \mid x, \\ \frac{2 v_{n}}{P_{n}} \cdot r_{m} a \mathcal{A}_{P_{m}} \approx \frac{v_{m}}{P_{m}} \cdot r_{m} a \mathcal{A}_{P_{n}}, & \text { if } P_{m} \mid x \text { and } P_{n} \backslash x, \\ \frac{v_{n}}{P_{n}} \cdot r_{m} a \mathcal{A}_{P_{m}} \approx \frac{2 v_{m}}{P_{m}} \cdot r_{m} a \mathcal{A}_{P_{n}}, & \text { if } P_{m} \backslash x \text { and } P_{n} \mid x, \\ \frac{2 v_{n}}{P_{n}} \cdot r_{m} a \mathcal{A}_{P_{m}} \approx \frac{2 v_{m}}{P_{m}} \cdot r_{m} a \mathcal{A}_{P_{n}}, & \text { if } P_{m} \backslash x \text { and } P_{n} \backslash \chi x .\end{cases}
$$

Therefore, if we sieve integers, then due to (5.8), at the $m^{\text {th }}$ round of the sieve, we have

$$
S\left(r_{m} \mathcal{A}_{P_{n}}, P_{m}\right) \approx r_{m} \mathcal{A}_{P_{n}} \cdot\left(\frac{P_{m}-v_{m}}{P_{m}}\right)
$$


ON THE ADDITIVE AND SUBTRACTIVE REPRESENTATION OF EVEN NUMBERS FROM PRIMES

and, if we sieve additive representations, then due to (5.20), at the $m^{\text {th }}$ round of the sieve, we have

$$
S\left(r_{m} a \mathcal{A}_{P_{n}}, P_{m}\right) \approx \begin{cases}r_{m} a \mathcal{A}_{P_{n}} \cdot\left(\frac{P_{m}-v_{m}}{P_{m}}\right), & \text { if } P_{m} \mid x, \\ r_{m} a \mathcal{A}_{P_{n}} \cdot\left(\frac{P_{m}-2 v_{m}}{P_{m}}\right), & \text { if } P_{m} \nmid x .\end{cases}
$$

Therefore, if prior to the $m^{\text {th }}$ round of the sieve, we have

$$
\frac{r_{m} a \mathcal{A}_{P_{n}}}{r_{m} a \mathcal{A}}=\frac{r_{m} \mathcal{A}_{P_{n}}}{r_{m} \mathcal{A}}
$$

then, due to (5.17), (5.18), (5.21), and (5.22), post the $m^{\text {th }}$ round of the sieve, we have

$$
\frac{r_{m+1} a \mathcal{A}_{P_{n}}}{r_{m+1} a \mathcal{A}} \approx \frac{r_{m+1} \mathcal{A}_{P_{n}}}{r_{m+1} \mathcal{A}} \text {. }
$$

Therefore, (5.4) is almost preserved prior to the $n^{\text {th }}$ round of the sieve, and thus we have

$$
\frac{r_{n} a \mathcal{A}_{P_{n}}}{r_{n} a \mathcal{A}} \approx \begin{cases}1 \cdot \frac{r_{n} \mathcal{A}_{P_{n}}}{r_{n} \mathcal{A}}, & \text { if } P_{n} \mid x, \\ 2 \cdot \frac{r_{n} \mathcal{A}_{P_{n}}}{r_{n} \mathcal{A}}, & \text { if } P_{n} \backslash x,\end{cases}
$$

and thus, we have generalised (3.23).

Due to (5.25), we have

$$
\frac{r_{n} a \mathcal{A}_{P_{n}}}{r_{n} a \mathcal{A}}= \begin{cases}1 \cdot \frac{r_{n} \mathcal{A}_{P_{n}}}{r_{n} \mathcal{A}} \cdot f_{a}, & \text { if } P_{n} \mid x, \\ 2 \cdot \frac{r_{n} \mathcal{A}_{P_{n}}}{r_{n} \mathcal{A}} \cdot f_{a}, & \text { if } P_{n} \backslash x,\end{cases}
$$

where $f_{a}$ is a positive real number. We determine an upper bound to $f_{a}$, as follows.

Since $P_{n} \leq \sqrt{x}$ and $r_{n} \mathcal{A} \geq \pi[\sqrt{x}, x]$, then

$$
\frac{P_{n}}{r_{n} \mathcal{A}} \leq \frac{\sqrt{x}}{\pi[\sqrt{x}, x]} .
$$

Since $\pi[\sqrt{x}, x] \rightarrow \pi(x)$ as $x \rightarrow \infty$, and

$$
\lim _{x \rightarrow \infty} \frac{\sqrt{x}}{\pi(x)}=0
$$

as implied by the Prime Number Theorem, then

$$
\lim _{x \rightarrow \infty} \frac{\sqrt{x}}{\pi[\sqrt{x}, x]}=0,
$$

and therefore

$$
\lim _{x \rightarrow \infty} \frac{P_{n}}{r_{n} \mathcal{A}}=0
$$

Therefore, if

$$
\frac{P_{n}}{r_{n} \mathcal{A}} \cdot r_{n} \mathcal{A}_{P_{n}}=c_{a}>0
$$


and therefore

$$
\frac{P_{n}}{r_{n} a \mathcal{A}} \cdot r_{n} a \mathcal{A}_{P_{n}} \approx c_{a}
$$

where $c_{a}$ is a constant, then $r_{n} \mathcal{A}_{P_{n}} \rightarrow \infty$ as $x \rightarrow \infty$, and therefore $r_{n} a \mathcal{A}_{P_{n}} \rightarrow \infty$ as $x \rightarrow \infty$. Therefore, if

$$
\frac{P_{n}}{r_{n} a \mathcal{A}} \cdot r_{n} a \mathcal{A}_{P_{n}} \geq c_{a},
$$

then $f_{a} \rightarrow 1$ as $x \rightarrow \infty$, and therefore, if $x \geq w$, then $f_{a} \leq 1.15$. Since $c_{a}$ may be chosen arbitrarily close to 0 , then

$$
\frac{r_{n} a \mathcal{A}_{P_{n}}}{r_{n} a \mathcal{A}} \leq \begin{cases}1 \cdot \frac{r_{n} \mathcal{A}_{P_{n}}}{r_{n} \mathcal{A}} \cdot 1.15, & \text { if } P_{n} \mid x \\ 2 \cdot \frac{r_{n} \mathcal{A}_{P_{n}}}{r_{n} \mathcal{A}} \cdot 1.15, & \text { if } P_{n} \backslash x\end{cases}
$$

Since

$$
\frac{r_{n} \mathcal{A}_{P_{n}}}{r_{n} \mathcal{A}} \leq \frac{2.15}{P_{n}}
$$

then

$$
\frac{r_{n} a \mathcal{A}_{P_{n}}}{r_{n} a \mathcal{A}} \leq \begin{cases}1 \cdot \frac{2.15}{P_{n}} \cdot 1.15, & \text { if } P_{n} \mid x, \\ 2 \cdot \frac{2.15}{P_{n}} \cdot 1.15, & \text { if } P_{n} \backslash x,\end{cases}
$$

and therefore

$$
\frac{r_{n} a \mathcal{A}_{P_{n}}}{r_{n} a \mathcal{A}} \leq \frac{5}{P_{n}}
$$

Furthermore, empirical data show that

$$
\frac{r_{1} a \mathcal{A}_{P_{1}}}{r_{1} a \mathcal{A}} \leq \frac{1}{P_{1}}
$$

and

$$
\frac{r_{2} a \mathcal{A}_{P_{2}}}{r_{2} a \mathcal{A}} \leq \frac{2}{P_{2}}+2
$$

and

$$
\frac{r_{3} \mathcal{A}_{P_{3}}}{r_{3} \mathcal{A}} \leq \frac{1}{P_{3}}+1
$$

Due to (5.34) and (5.40), we have

$$
\frac{r_{3} a \mathcal{A}_{P_{3}}}{r_{3} a \mathcal{A}} \leq 2 \cdot\left(\frac{1}{P_{3}}+1\right) \cdot 1.15<\frac{3}{P_{3}}+3 .
$$

With (5.37), (5.38), (5.39), and (5.41), we have determined upper bounds to (3.18), on the condition that $x \geq w$. 


\section{UPPER BOUNDS ON THE SIFTED OUT SUBTRACTIVE REPRESENTATIONS}

In this section, we determine upper bounds to (3.30), as follows. Prior to the $1^{\text {st }}$ round of the sieve, we have

$$
r_{1} S \mathcal{A}=\frac{1}{2} \cdot r_{1} \mathcal{A}^{\prime}
$$

and

$$
\begin{cases}\text { if } P_{n} \mid 2 k \text { and } P_{n} \mid p, & \text { then } P_{n} \mid(p+2 k), \\ \text { if } P_{n} \backslash 2 k \text { and } P_{n} \mid p, & \text { then } P_{n} \backslash(p+2 k), \\ \text { if } P_{n} \backslash 2 k \text { and } P_{n} \mid(p+2 k), & \text { then } P_{n} \backslash p,\end{cases}
$$

and therefore

$$
r_{1} s \mathcal{A}_{P_{n}}= \begin{cases}\frac{1}{2} \cdot r_{1} \mathcal{A}_{P_{n}}^{\prime}, & \text { if } P_{n} \mid 2 k \\ \frac{1}{1} \cdot r_{1} \mathcal{A}_{P_{n}}^{\prime}, & \text { if } P_{n} \backslash 2 k\end{cases}
$$

Due to (6.1) and (6.3), we have

$$
\frac{r_{1} s \mathcal{A}_{P_{n}}}{r_{1} S \mathcal{A}}= \begin{cases}1 \cdot \frac{r_{1} \mathcal{A}_{P_{n}}^{\prime}}{r_{1} \mathcal{A}^{\prime}}, & \text { if } P_{n} \mid 2 k, \\ 2 \cdot \frac{r_{1} \mathcal{A}_{P_{n}}^{\prime}}{r_{1} \mathcal{A}^{\prime}}, & \text { if } P_{n} \backslash 2 k\end{cases}
$$

If $2 k \leq \frac{x}{2}$, then

$$
\frac{r_{1} \mathcal{A}_{P_{n}}^{\prime}}{r_{1} \mathcal{A}^{\prime}}=\frac{r_{1} \mathcal{A}_{P_{n}}}{r_{1} \mathcal{A}}
$$

and therefore

$$
\frac{r_{1} s \mathcal{A}_{P_{n}}}{r_{1} s \mathcal{A}}= \begin{cases}1 \cdot \frac{r_{1} \mathcal{A}_{P_{n}}}{r_{1} \mathcal{A}}, & \text { if } P_{n} \mid 2 k \\ 2 \cdot \frac{r_{1} \mathcal{A}_{P_{n}}}{r_{1} \mathcal{A}}, & \text { if } P_{n} \backslash 2 k\end{cases}
$$

In the following, we describe the sieving process of subtractive representations at the $m^{\text {th }}$ round of the sieve, in comparison to the sieving process of integers, also at the $m^{\text {th }}$ round of the sieve. The $m^{\text {th }}$ round of the sieve precedes the $n^{\text {th }}$ round of the sieve. Thereby, we show that (6.6) is almost preserved prior to the $n^{\text {th }}$ round of the sieve, hence generalising (3.35).

Prior to the $m^{\text {th }}$ round of the sieve, we have

$$
r_{m} \mathcal{A}_{P_{m}}^{\prime}=\frac{t_{m}}{P_{m}} \cdot r_{m} \mathcal{A}^{\prime},
$$

and

$$
r_{m} \mathcal{A}_{P_{n}}^{\prime}=\frac{t_{n}}{P_{n}} \cdot r_{m} \mathcal{A}^{\prime}
$$

and therefore

$$
r_{m} \mathcal{A}_{P_{m} P_{n}}^{\prime}=\frac{t_{m}}{P_{m}} \cdot \frac{t_{n}}{P_{n}} \cdot r_{m} \mathcal{A}^{\prime},
$$


and therefore

$$
\frac{t_{n}}{P_{n}} \cdot r_{m} \mathcal{A}_{P_{m}}^{\prime} \approx \frac{t_{m}}{P_{m}} \cdot r_{m} \mathcal{A}_{P_{n}}^{\prime}
$$

When sieving subtractive representations, prior to the $n^{\text {th }}$ round of the sieve, we have

$$
\begin{cases}\text { if } p \in r_{n} s \mathcal{A}, & \text { then }(p+2 k) \in r_{n} s \mathcal{A}, \\ \text { if }(p+2 k) \in r_{n} s \mathcal{A}, & \text { then } p \in r_{n} s \mathcal{A},\end{cases}
$$

and therefore, we assume that

$$
\begin{cases}\text { if } p \in r_{m} \mathcal{A}^{\prime}, & \text { then }(p+2 k) \in r_{m} \mathcal{A}^{\prime}, \\ \text { if }(p+2 k) \in r_{m} \mathcal{A}^{\prime}, & \text { then } p \in r_{m} \mathcal{A}^{\prime} .\end{cases}
$$

Thus, we have

$$
r_{m} s \mathcal{A}=\frac{1}{2} \cdot r_{m} \mathcal{A}^{\prime}
$$

and

$$
\begin{cases}\text { if } P_{m} \mid 2 k \text { and } P_{m} \mid p, & \text { then } P_{m} \mid(p+2 k), \\ \text { if } P_{m} \backslash 2 k \text { and } P_{m} \mid p, & \text { then } P_{m} \backslash(p+2 k), \\ \text { if } P_{m} \backslash 2 k \text { and } P_{m} \mid(p+2 k), & \text { then } P_{m} \backslash p,\end{cases}
$$

and therefore

$$
r_{m} s \mathcal{A}_{P_{m}}= \begin{cases}\frac{1}{2} \cdot r_{m} \mathcal{A}_{P_{m}}^{\prime}, & \text { if } P_{m} \mid 2 k \\ \frac{1}{1} \cdot r_{m} \mathcal{A}_{P_{m}}^{\prime}, & \text { if } P_{m} \backslash 2 k\end{cases}
$$

and

$$
\begin{cases}\text { if } P_{n} \mid 2 k \text { and } P_{n} \mid p, & \text { then } P_{n} \mid(p+2 k), \\ \text { if } P_{n} \backslash 2 k \text { and } P_{n} \mid p, & \text { then } P_{n} \backslash(p+2 k), \\ \text { if } P_{n} \backslash 2 k \text { and } P_{n} \mid(p+2 k), & \text { then } P_{n} \backslash p,\end{cases}
$$

and therefore

$$
r_{m} s \mathcal{A}_{P_{n}}= \begin{cases}\frac{1}{2} \cdot r_{m} \mathcal{A}_{P_{n}}^{\prime}, & \text { if } P_{n} \mid 2 k, \\ \frac{1}{1} \cdot r_{m} \mathcal{A}_{P_{n}}^{\prime}, & \text { if } P_{n} \backslash 2 k .\end{cases}
$$

Due to (6.7), (6.13), and (6.15), we have

$$
r_{m} s \mathcal{A}_{P_{m}}= \begin{cases}\frac{t_{m}}{P_{m}} \cdot r_{m} s \mathcal{A}, & \text { if } P_{m} \mid 2 k, \\ \frac{2 t_{m}}{P_{m}} \cdot r_{m} s \mathcal{A}, & \text { if } P_{m} \backslash 2 k .\end{cases}
$$

Therefore, if we sieve integers, then due to (6.7), at the $m^{\text {th }}$ round of the sieve, we have

$$
S\left(r_{m} \mathcal{A}^{\prime}, P_{m}\right)=r_{m} \mathcal{A}^{\prime} \cdot\left(\frac{P_{m}-t_{m}}{P_{m}}\right)
$$


ON THE ADDITIVE AND SUBTRACTIVE REPRESENTATION OF EVEN NUMBERS FROM PRIMES

and, if we sieve subtractive representations, then due to (6.18), at the $m^{\text {th }}$ round of the sieve, we have

$$
S\left(r_{m} s \mathcal{A}, P_{m}\right)= \begin{cases}r_{m} s \mathcal{A} \cdot\left(\frac{P_{m}-t_{m}}{P_{m}}\right), & \text { if } P_{m} \mid 2 k, \\ r_{m} s \mathcal{A} \cdot\left(\frac{P_{m}-2 t_{m}}{P_{m}}\right), & \text { if } P_{m} \backslash 2 k .\end{cases}
$$

Furthermore, due to (6.7), (6.8), (6.13), (6.15), and (6.17), we have

$$
r_{m} s \mathcal{A}_{P_{m} P_{n}}= \begin{cases}\frac{t_{m}}{P_{m}} \cdot \frac{t_{n}}{P_{n}} \cdot r_{m} s \mathcal{A}, & \text { if } P_{m} \mid 2 k \text { and } P_{n} \mid 2 k, \\ \frac{t_{m}}{P_{m}} \cdot \frac{2 t_{n}}{P_{n}} \cdot r_{m} s \mathcal{A}, & \text { if } P_{m} \mid 2 k \text { and } P_{n} \backslash 2 k, \\ \frac{2 t_{m}}{P_{m}} \cdot \frac{t_{n}}{P_{n}} \cdot r_{m} s \mathcal{A}, & \text { if } P_{m} \backslash 2 k \text { and } P_{n} \mid 2 k, \\ \frac{2 t_{m}}{P_{m}} \cdot \frac{2 t_{n}}{P_{n}} \cdot r_{m} s \mathcal{A}, & \text { if } P_{m} \backslash 2 k \text { and } P_{n} \backslash 2 k,\end{cases}
$$

and therefore

$$
r_{m} s \mathcal{A}_{P_{m} P_{n}} \approx \begin{cases}\frac{t_{n}}{P_{n}} \cdot r_{m} s \mathcal{A}_{P_{m}} \approx \frac{t_{m}}{P_{m}} \cdot r_{m} s \mathcal{A}_{P_{n}}, & \text { if } P_{m} \mid 2 k \text { and } P_{n} \mid 2 k, \\ \frac{2 t_{n}}{P_{n}} \cdot r_{m} s \mathcal{A}_{P_{m}} \approx \frac{t_{m}}{P_{m}} \cdot r_{m} s \mathcal{A}_{P_{n}}, & \text { if } P_{m} \mid 2 k \text { and } P_{n} \backslash 2 k, \\ \frac{t_{n}}{P_{n}} \cdot r_{m} s \mathcal{A}_{P_{m}} \approx \frac{2 t_{m}}{P_{m}} \cdot r_{m} s \mathcal{A}_{P_{n}}, & \text { if } P_{m} \backslash 2 k \text { and } P_{n} \mid 2 k, \\ \frac{2 t_{n}}{P_{n}} \cdot r_{m} s \mathcal{A}_{P_{m}} \approx \frac{2 t_{m}}{P_{m}} \cdot r_{m} s \mathcal{A}_{P_{n}}, & \text { if } P_{m} \backslash 2 k \text { and } P_{n} \backslash 2 k\end{cases}
$$

Therefore, if we sieve integers, then due to (6.10), at the $m^{\text {th }}$ round of the sieve, we have

$$
S\left(r_{m} \mathcal{A}_{P_{n}}^{\prime}, P_{m}\right) \approx r_{m} \mathcal{A}_{P_{n}}^{\prime} \cdot\left(\frac{P_{m}-t_{m}}{P_{m}}\right)
$$

and, if we sieve subtractive representations, then due to (6.22), at the $m^{\text {th }}$ round of the sieve, we have

$$
S\left(r_{m} s \mathcal{A}_{P_{n}}, P_{m}\right) \approx \begin{cases}r_{m} s \mathcal{A}_{P_{n}} \cdot\left(\frac{P_{m}-t_{m}}{P_{m}}\right), & \text { if } P_{m} \mid 2 k, \\ r_{m} S \mathcal{A}_{P_{n}} \cdot\left(\frac{P_{m}-2 t_{m}}{P_{m}}\right), & \text { if } P_{m} \curlywedge 2 k .\end{cases}
$$

Therefore, if prior to the $m^{\text {th }}$ round of the sieve, we have

$$
\frac{r_{m} s \mathcal{A}_{P_{n}}}{r_{m} s \mathcal{A}}=\frac{r_{m} \mathcal{A}_{P_{n}}^{\prime}}{r_{m} \mathcal{A}^{\prime}},
$$

then, due to (6.19), (6.20), (6.23), and (6.24), post the $m^{\text {th }}$ round of the sieve, we have

$$
\frac{r_{m+1} s \mathcal{A}_{P_{n}}}{r_{m+1} S \mathcal{A}} \approx \frac{r_{m+1} \mathcal{A}_{P_{n}}^{\prime}}{r_{m+1} \mathcal{A}^{\prime}} .
$$


Therefore, (6.4) is almost preserved prior to the $n^{\text {th }}$ round of the sieve, and thus we have

$$
\frac{r_{n} s \mathcal{A}_{P_{n}}}{r_{n} s \mathcal{A}} \approx \begin{cases}1 \cdot \frac{r_{n} \mathcal{A}_{P_{n}}^{\prime}}{r_{n} \mathcal{P}^{\prime}}, & \text { if } P_{n} \mid 2 k, \\ 2 \cdot \frac{r_{n} \mathcal{A}_{P_{n}}^{\prime}}{r_{n} \mathcal{P}^{\prime}}, & \text { if } P_{n} \backslash 2 k .\end{cases}
$$

If $2 k \leq \frac{x}{2}$, then

$$
\frac{r_{n} \mathcal{A}_{P_{n}}^{\prime}}{r_{n} \mathcal{A}^{\prime}}=\frac{r_{n} \mathcal{A}_{P_{n}}}{r_{n} \mathcal{A}},
$$

and therefore

$$
\frac{r_{n} s \mathcal{A}_{P_{n}}}{r_{n} s \mathcal{A}} \approx \begin{cases}1 \cdot \frac{r_{n} \mathcal{A}_{P_{n}}}{r_{n} \mathcal{A}}, & \text { if } P_{n} \mid 2 k, \\ 2 \cdot \frac{r_{n} \mathcal{A}_{P_{n}}}{r_{n} \mathcal{A}}, & \text { if } P_{n} \backslash 2 k,\end{cases}
$$

and thus, we have generalised (3.35).

Due to (6.29), we have

$$
\frac{r_{n} s \mathcal{A}_{P_{n}}}{r_{n} s \mathcal{A}}= \begin{cases}1 \cdot \frac{r_{n} \mathcal{A}_{P_{n}}}{r_{n} \mathcal{A}} \cdot f_{s}, & \text { if } P_{n} \mid 2 k, \\ 2 \cdot \frac{r_{n} \mathcal{A}_{P_{n}}}{r_{n} \mathcal{A}} \cdot f_{s}, & \text { if } P_{n} \backslash 2 k,\end{cases}
$$

where $f_{s}$ is a positive real number. We determine an upper bound to $f_{s}$, as follows. Since $P_{n} \leq \sqrt{x}$ and $r_{n} \mathcal{A} \geq \pi[\sqrt{x}, x]$, then

$$
\frac{P_{n}}{r_{n} \mathcal{A}} \leq \frac{\sqrt{x}}{\pi[\sqrt{x}, x]} .
$$

Since $\pi[\sqrt{x}, x] \rightarrow \pi(x)$ as $x \rightarrow \infty$, and

$$
\lim _{x \rightarrow \infty} \frac{\sqrt{x}}{\pi(x)}=0,
$$

as implied by the Prime Number Theorem, then

$$
\lim _{x \rightarrow \infty} \frac{\sqrt{x}}{\pi[\sqrt{x}, x]}=0,
$$

and therefore

$$
\lim _{x \rightarrow \infty} \frac{P_{n}}{r_{n} \mathcal{A}}=0
$$

Therefore, if

$$
\frac{P_{n}}{r_{n} \mathcal{A}} \cdot r_{n} \mathcal{A}_{P_{n}}=c_{s}>0,
$$

and therefore

$$
\frac{P_{n}}{r_{n} s \mathcal{A}} \cdot r_{n} s \mathcal{A}_{P_{n}} \approx c_{s},
$$


ON THE ADDITIVE AND SUBTRACTIVE REPRESENTATION OF EVEN NUMBERS FROM PRIMES

where $c_{s}$ is a constant, then $r_{n} \mathcal{A}_{P_{n}} \rightarrow \infty$ as $x \rightarrow \infty$, and therefore $r_{n} s \mathcal{A}_{P_{n}} \rightarrow \infty$ as $x \rightarrow \infty$. Therefore, if

$$
\frac{P_{n}}{r_{n} s \mathcal{A}} \cdot r_{n} s \mathcal{A}_{P_{n}} \geq c_{s}
$$

then $f_{s} \rightarrow 1$ as $x \rightarrow \infty$, and therefore, if $x \geq w$, then $f_{s} \leq 1.15$. Since $c_{s}$ may be chosen arbitrarily close to 0 , then

$$
\frac{r_{n} s \mathcal{A}_{P_{n}}}{r_{n} s \mathcal{A}} \leq \begin{cases}1 \cdot \frac{r_{n} \mathcal{A}_{P_{n}}}{r_{n} \mathcal{A}} \cdot 1.15, & \text { if } P_{n} \mid 2 k \\ 2 \cdot \frac{r_{n} \mathcal{A}_{P_{n}}}{r_{n} \mathcal{A}} \cdot 1.15, & \text { if } P_{n} \backslash 2 k\end{cases}
$$

Since

$$
\frac{r_{n} \mathcal{A}_{P_{n}}}{r_{n} \mathcal{A}} \leq \frac{2.15}{P_{n}}
$$

then

$$
\frac{r_{n} s \mathcal{A}_{P_{n}}}{r_{n} s \mathcal{A}} \leq \begin{cases}1 \cdot \frac{2.15}{P_{n}} \cdot 1.15, & \text { if } P_{n} \mid 2 k \\ 2 \cdot \frac{2.15}{P_{n}} \cdot 1.15, & \text { if } P_{n} \backslash 2 k\end{cases}
$$

and therefore

$$
\frac{r_{n} s \mathcal{A}_{P_{n}}}{r_{n} s \mathcal{A}} \leq \frac{5}{P_{n}}
$$

Furthermore, empirical data show that

$$
\frac{r_{1} s \mathcal{A}_{P_{1}}}{r_{1} s \mathcal{A}} \leq \frac{1}{P_{1}}
$$

and

$$
\frac{r_{2} s \mathcal{A}_{P_{2}}}{r_{2} s \mathcal{A}} \leq \frac{2}{P_{2}}+2
$$

and

$$
\frac{r_{3} \mathcal{A}_{P_{3}}}{r_{3} \mathcal{A}} \leq \frac{1}{P_{3}}+1
$$

Due to (6.38) and (6.44), we have

$$
\frac{r_{3} S \mathcal{A}_{P_{3}}}{r_{3} s \mathcal{A}} \leq 2 \cdot\left(\frac{1}{P_{3}}+1\right) \cdot 1.15<\frac{3}{P_{3}}+3 .
$$

With (6.41), (6.42), (6.43), and (6.45), we have determined upper bounds to (3.30), on the condition that $x \geq w$.

\section{LOWER BOUNDS ON THE SURVIVING REPRESENTATIONS}

Let $x \geq w$, then due to the upper bounds in (5.37), (5.38), (5.39), and (5.41), we have the following lower bound for (3.22):

$$
g(x)^{\prime} \geq\left(\frac{x}{2} \cdot \frac{\left(P_{1}-1\right)}{P_{1}} \cdot \frac{\left(P_{2}-2\right)}{P_{2}} \cdot \frac{\left(P_{3}-3\right)}{P_{3}} \cdot \prod_{P_{n}=P_{4}}^{P_{n} \leq \sqrt{x}} \frac{\left(P_{n}-5\right)}{P_{n}}\right)-6,
$$


which we quantify as follows. Since

$$
\prod_{P_{n}=P_{4}}^{P_{n} \leq \sqrt{x}} \frac{\left(P_{n}-5\right)}{P_{n}} \geq \prod_{P_{n}=P_{4}}^{P_{n}=P_{70}} \frac{\left(P_{n}-5\right)}{P_{n}} \cdot \prod_{P_{n}=P_{71}}^{P_{n} \leq \sqrt{x}} \frac{\left(P_{n}-6\right)}{P_{n}},
$$

and

$$
\frac{\left(P_{1}-1\right)}{P_{1}} \cdot \frac{\left(P_{2}-2\right)}{P_{2}} \cdot \frac{\left(P_{3}-3\right)}{P_{3}} \cdot \prod_{P_{n}=P_{4}}^{P_{n}=P_{70}} \frac{\left(P_{n}-5\right)}{P_{n}}>\frac{79}{500,000}
$$

then

$$
g(x)^{\prime} \geq\left(x \cdot \frac{79}{1,000,000} \cdot \prod_{P_{n}=P_{71}}^{P_{n} \leq \sqrt{x}} \frac{\left(P_{n}-6\right)}{P_{n}}\right)-6 .
$$

Since $P_{71}=O_{118}$, then

$$
\prod_{P_{n}=P_{71}}^{P_{n} \leq \sqrt{x}} \frac{\left(P_{n}-6\right)}{P_{n}} \geq \prod_{O_{z}=O_{118}}^{O_{z} \leq \sqrt{x}} \frac{\left(O_{z}-6\right)}{O_{z}}
$$

and therefore

$$
g(x)^{\prime} \geq\left(x \cdot \frac{79}{1,000,000} \cdot \prod_{O_{z}=O_{118}}^{O_{z} \leq \sqrt{x}} \frac{\left(O_{z}-6\right)}{O_{z}}\right)-6 .
$$

Since $O_{z} \leq \sqrt{x}$, then

$$
\prod_{O_{z}=O_{118}}^{O_{z} \leq \sqrt{x}} \frac{\left(O_{z}-6\right)}{O_{z}} \geq \frac{O_{116} \cdot O_{117}}{x}
$$

Since $O_{116}=347$ and $O_{117}=349$, then

$$
g(x)^{\prime} \geq\left(\frac{79 \cdot 347 \cdot 349}{1,000,000}\right)-6 \geq 1 .
$$

This completes the proof for theorem 1.

Let $x \geq w$ and let $2 k \leq \frac{x}{2}$, then due to the upper bounds in (6.41), (6.42), (6.43), and (6.45), we have the following lower bound for (3.34):

$$
\pi_{2 k}(x)^{\prime} \geq\left(\frac{x}{2} \cdot \frac{\left(P_{1}-1\right)}{P_{1}} \cdot \frac{\left(P_{2}-2\right)}{P_{2}} \cdot \frac{\left(P_{3}-3\right)}{P_{3}} \cdot \prod_{P_{n}=P_{4}}^{P_{n} \leq \sqrt{x}} \frac{\left(P_{n}-5\right)}{P_{n}}\right)-6 .
$$

Therefore, as with (7.8), we have

$$
\pi_{2 k}(x)^{\prime} \geq\left(\frac{79 \cdot 347 \cdot 349}{1,000,000}\right)-6 \geq 1,
$$

and therefore $\pi_{2 k}(x) \rightarrow \infty$ as $x \rightarrow \infty$. This completes the proof for theorem 2 . 


\section{ACKNOWLEDGEMENTS}

In constructing the proofs presented in this paper, we were constantly guided by observations of empirical data, such as those presented in section 3. To this end, we give very special thanks to our dear friends, Abdul Karim and Zhuen Xie, with the assistance of whom we were able to obtain the data in a much shorter time frame than we would have otherwise. We're also very grateful for the free access to the following websites, which we've made extensive use of: oeis.org/and onlinenumbertools.com/calculate-prime-factors

\section{REFERENCES}

[1] L. E. Dickson. Goldbach's Empirical Theorem: Every Integer is a Sum of Two Primes, in History of the Theory of Numbers, Vol. 1: Divisibility and Primality. Dover Publications, New York, 1952, pp. 421-424.

[2] R. K. Guy. Goldbach's conjecture, in Unsolved Problems in Number Theory. Springer-Verlag, New York, 2004, pp. 159-164.

[3] T. O. Silva, S. Herzog, and S. Pard. Empirical verification of the even Goldbach conjecture and computation of prime gaps up to $4 \cdot 10^{18}$. Math. Comp. 83 (2014), 2033-2060.

[4] V. Brun. The Sieve of Eratosthenes and the Theorem of Goldbach, in Goldbach Conjecture. World Scientific, Singapore, 2002, pp. 99-136.

[5] H. L. Montgomery and R. C. Vaughan. The exceptional set in Goldbach's problem. in Acta. Arith. 27 (1975), 353-370.

[6] O. Ramare. On Šnirel'man's constant. Ann. Scuola. Norm. Sup. Pis. 22 (1995), 645-706.

[7] J. R. Chen. On the representation of a larger even integer as the sum of a prime and the product of at most two primes. Sci. Sinica. 16 (1973), 157-176.

[8] I. M. Vinogradov. Representations of an odd integer as a sum of three primes. in Goldbach Conjecture. World Scientific, Singapore, 2002, pp. 61-64.

[9] T. Tao. Every odd number greater than 1 is the sum of at most five primes. Preprint, $\operatorname{arxiv.org/abs/1201.}$ 6656, July 2012.

[10] H. Helfgott. The ternary Goldbach conjecture is true. Preprint, arxiv.org/abs/1312.7748, December 2013.

[11] L. E. Dickson. Theorems Analogous to Goldbach's. in History of the Theory of Numbers, Vol. 1: Divisibility and Primality. Dover Publications, New York, 1952, pp. 424-425.

[12] H. Rezgui. Conjecture of twin primes (Still unsolved problem in Number Theory). An expository essay. Sur. Math. App. 12 (2017), 229-252.

[13] L. Bethune. Found: New World Record Twin Primes!, 2016: available at: https://www.epcc.ed.ac.uk/ blog/2016/09/27/found-new-world-record-twin-prime

[14] D. A. Goldston, J. Pintz, and C. Y. Yıldırım. Primes in tuples. I. Ann. of Math. 170, No. 2, (2009), 819-862.

[15] Y. Zhang. Bounded gaps between primes. Ann. Math. 179 (2014), 1121-1174.

[16] J. Maynard. Small gaps between primes. Ann. Math. 181 (2015), 1-31.

[17] D. H. J. Polymath. The "bounded gaps between primes" Polymath project - a retrospective. Preprint, arxiv . org/abs/1409.8361, September 2014.

[18] S. Horsley. The Sieve of Eratosthenes. Being an account of his method of finding all the Prime Numbers. Phil. Trans. 62 (1772), 327-347. 